This item was submitted to Loughborough's Research Repository by the author.

Items in Figshare are protected by copyright, with all rights reserved, unless otherwise indicated.

\title{
A consideration of methods of determining the radiative characteristics of jet
} fires

PLEASE CITE THE PUBLISHED VERSION

http://dx.doi.org/10.1016/j.combustflame.2011.09.004

PUBLISHER

(c) Elsevier Inc.

VERSION

AM (Accepted Manuscript)

LICENCE

CC BY-NC-ND 4.0

REPOSITORY RECORD

Hankinson, Geoffrey, and Barbara J. Lowesmith. 2014. "A Consideration of Methods of Determining the Radiative Characteristics of Jet Fires". figshare. https://hdl.handle.net/2134/15056. 
This item was submitted to Loughborough's Institutional Repository (https://dspace.lboro.ac.uk/) by the author and is made available under the following Creative Commons Licence conditions.

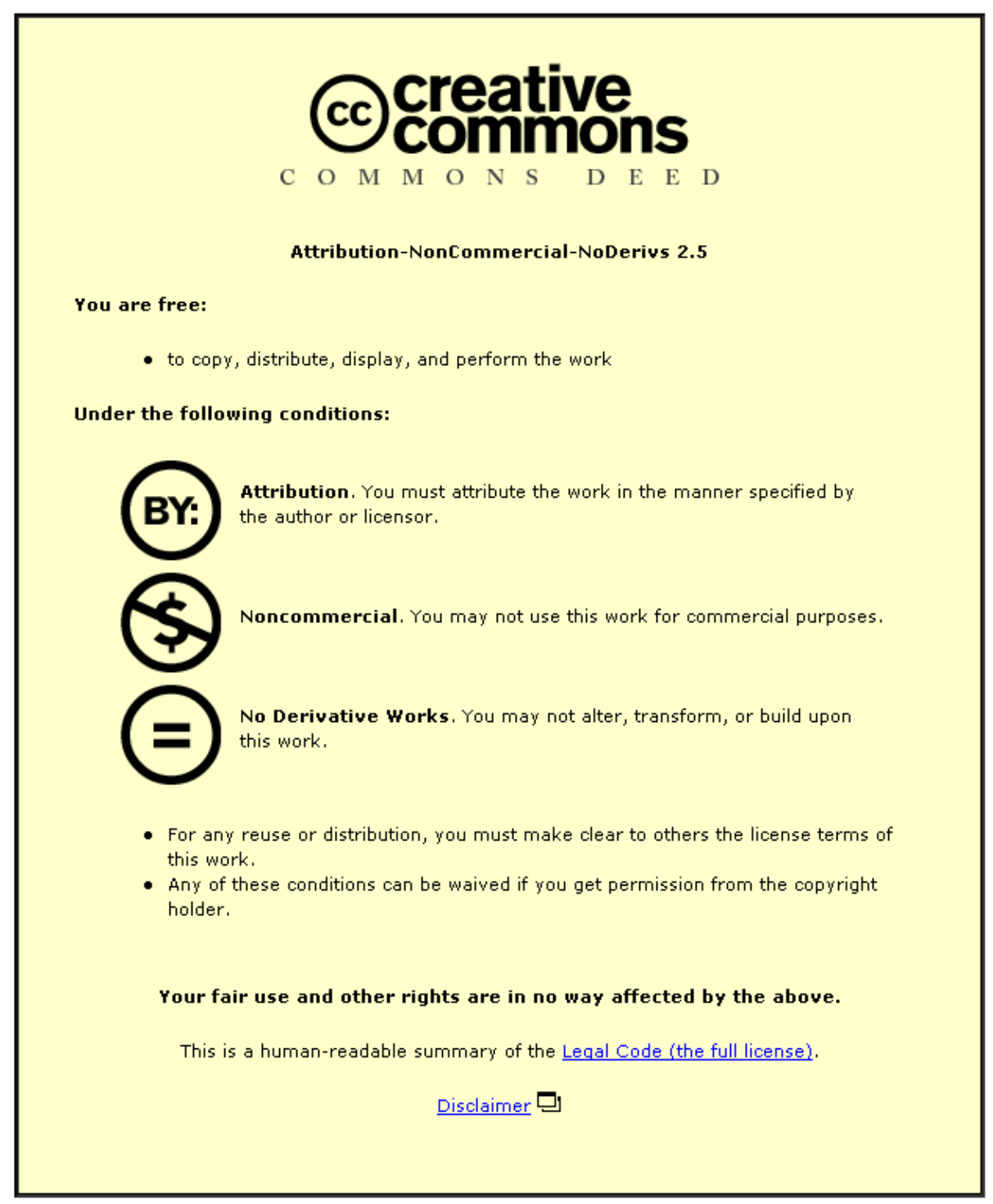

For the full text of this licence, please go to: http://creativecommons.org/licenses/by-nc-nd/2.5/ 


\title{
A CONSIDERATION OF METHODS OF DETERMINING THE RADIATIVE CHARACTERISTICS OF JET FIRES
}

\author{
G. HANKINSON ${ }^{1}$ and B. J. LOWESMITH ${ }^{1}$ \\ ${ }^{1}$ Loughborough University, Loughborough, UK
}

Abstract

The radiative characteristics of jet fires is usually expressed through the use of a fraction of heat radiated, which is primarily a property of the fuel being considered. It is generally determined from experimental data of incident radiation around a fire and then derived by using a model of the incident radiation in terms of the fraction of heat radiated. Popular approaches include the single point source model where the flame is represented by a single point usually located halfway along the flame, or use of an idealised flame shape, such as a cylinder or cone, and deriving the flame surface emissive power which is closely related to the fraction of heat radiated. However, these modelling approaches may provide erroneous results for the fraction of heat radiated if incident radiation data in the near-field is used, and the fraction of heat radiated derived using one modelling approach may not be applicable to another approach without some adjustment. This paper explores the inherent near-field and far-field behaviour of different modelling approaches and the resulting impact on the fraction of heat radiated derived from each modelling approach using incident radiation data. A weighted multi-point source approach model was found to replicate both near-field and far-field behaviour well and capable of deriving the true 
fraction of heat radiated. Four idealised shapes were considered and it was found that the true fraction of heat radiated would need to be adjusted for use with these models even in the far-field, and some shortcomings in near-field behaviour were identified, which would suggest that some weighting of the surface emissive power over different regions of the flame would be needed. Finally, an idealised shape with hemispherical point sources distributed over its surface was considered and this model behaved well in both the nearfield and far-field.

Keywords: jet fires; fraction of heat radiated; modelling thermal radiation

\section{BACKGROUND}

The ability to determine incident radiation from large jet fires is essential for the safety of operations in the oil and gas industry, both from accidental releases which could form a jet fire on ignition and for the design of flare systems for use during upset conditions, allowing relief valves and blowdown systems to discharge safely.

The thermal radiation emitted by hydrocarbon jet fires comes, predominantly from two sources: spectrally continuous emission from burning soot particles and spectrally banded emission from hot combustion products such as $\mathrm{H}_{2} \mathrm{O}$ and $\mathrm{CO}_{2}$. Both emit mostly in the infra-red region but the continuous emission from hot soot particles extends to the visible spectrum and is responsible for the luminosity of flames. Hydrogen combustion cannot form soot or $\mathrm{CO}_{2}$ so the thermal emissions come entirely from hot water vapour. 
Industry requires engineering type calculation methods for the assessment of many potential accidental release scenarios, and to assist in the design of flare systems. One such simple calculation technique is the single point source approach, where the flame is approximated by a single point at its centre, although it is well known to have shortcomings for calculations in the near-field (typically within about one flame length). Alternatively, flames can be represented by an idealised shape, but again may have difficulties in predicting near-field radiation. All of these modelling approaches need some measure of the radiative properties of the flame itself, referred to as the fraction of heat radiated. This fraction of heat radiated is generally considered to be a characteristic of the fuel concerned but may also vary with other parameters, and it is usually derived from experimental data. Values of fraction of heat radiated can be found in the literature for common hydrocarbons, but if detailed explanation of their derivation is not provided, care needs to be taken regarding their applicability.

\section{INTRODUCTION}

Against this background, this paper attempts to explore the significance of the derivation of the fraction of heat radiated on the applicability of its use. That is to say, can values of fraction of heat radiated derived from data using one method, be applied for use within another model of the radiative characteristics of a fire, and if so, is some adjustment required? Various modelling approaches for jet fires are considered and by comparing their behaviour against data gathered in the near-field and far-field, the fundamental characteristics of the modelling approach are examined. This provides an indication of the 
potential suitability of a modelling approach to determine both near-field and far-field radiation using a value of fraction of heat radiated which is applicable to all distances from, and locations around, a fire. As will be seen later, what is meant by 'near-field' or 'farfield' may depend upon the position of the point of interest in the direction of the flame axis, whereby the near-field extends further for axial positions close to and beyond the beginning and end of the flame. Nevertheless, as a minimum, the near-field can be thought of as within about one flame length of the fire.

The application of the various modelling approaches to predict radiation from large scale jet fires is also considered and the dangers of extrapolation from small scale scenarios highlighted.

\section{FRACTION OF HEAT RADIATED}

The proportion of the energy of combustion which is released to the atmosphere as radiant energy is termed $Q_{r}(\mathrm{~kW})$, where $Q(k W)$ is the total power of combustion. The fraction of heat radiated is defined as:

$$
F=\frac{Q_{r}}{Q}
$$

where $Q=m H$ with $m$ being the mass flowrate $\left(\mathrm{kg} \mathrm{s}^{-1}\right)$ and $H$ being the net calorific value of the fuel $\left(\mathrm{kJ} \mathrm{kg}^{-1}\right)$. Hence if $Q_{r}$ can be measured, or inferred from measurements of incident radiation outside the fire, then $F$ can be determined from experimental data. In 
fact, this is the usual course of action. This means that the derivation of $F$ will be dependent upon the approach used to calculate the incident radiation outside the flame as a function of the relevant parameters, such as, fuel type, distance of the measurement from the fire, atmospheric conditions and fuel flowrate. This is an important point which is considered in some detail later in this paper. Some correlations for $F$ also include other parameters, in particular, those related to the geometry of the fire or the fuel release conditions. Nevertheless, it is generally accepted that the fraction of heat radiated is fuel specific, and some workers regard the value to be constant for each fuel over a range of release conditions (Kent [1]; Tan [2]; Lowesmith et al [3] (for sonic releases)). However, others have developed correlations which result in $F$ varying with parameters related to the jet exit velocity and/or fire geometry. For example, Cook et al [4] who related the fraction of heat radiated for methane to the exit velocity by:

$F=0.321-0.418 \times 10^{-3} u_{j}$

where $u_{j}$ is the exit velocity of the gas jet $\left(\mathrm{m} \mathrm{s}^{-1}\right)$. Similarly, Chamberlain et al [5] suggested:

$F=0.21 \mathrm{e}^{-0.00323 \mathrm{u}_{\mathrm{eq}}}+0.11$

where $u_{e q}$ is the effective exit velocity of the gas jet given by Birch et al [6], as: $u_{e q}=$ $u_{j}+\left(p_{j}-p_{\infty}\right) /\left(\rho_{j} u_{j}\right)$ where $p_{j}$ and $p_{\infty}$ are the absolute pressures at the jet exit pressure and in the atmosphere respectively; $\rho_{j}$ is the jet exit density. 
Other workers have related the fraction of heat radiated to the flame residence time, $t_{f}(\mathrm{~s})$, of the flame (Turns and Myhr [7]) which itself is a function of the release conditions and the flame dimensions, given by:

$t_{f}=\left(\rho_{f} W_{f}^{2} L_{f} y_{s}\right) /\left(3 \rho_{j} d_{j}^{2} u_{j}\right)$

where $\rho_{f}$ is the flame density $\left(\mathrm{kg} \mathrm{m}^{-3}\right)$ and is given by $p_{\infty} M /\left(R_{u} T_{a d}\right)$ (Houf and Schefer [8]), with $M$ being the molecular weight $\left(\mathrm{kg} \mathrm{kmol}^{-1}\right) ; R_{u}$ is the Universal Gas Constant (8314.3 $\mathrm{J} \mathrm{kmol}^{-1} \mathrm{~K}^{-1}$ ); $T_{a d}$ is the adiabatic flame temperature (K). $W_{f}$ and $L_{f}$ are the width and length of the visible flame (m); $y_{s}$ is the mass fraction of fuel at stoichiometric conditions; $d_{j}$ is the jet exit diameter (m). Molina et al [9] proposed:

$F=0.085 \log _{10}\left(t_{f} a_{f} T_{a d}^{4}\right)-1.16$

where $a_{f}$ is the Planck mean absorption coefficient $\left(\mathrm{m}^{-1}\right)$. Studer et al [10] noted that Eqs. (3) and (5) can result in significantly different values for $F$ and that care must be taken with regard to the applicability of these correlations. This is hardly surprising, since all the above correlations for $F$ arise from using a model of the incident radiation field around a jet fire together with experimentally measured values of the incident radiation to backcalculate the value of $F$ for a given fuel and release conditions, in order to provide agreement between the measured and predicted values. 
Therefore, $F$ is inherently a function of the model of incident radiation by which it was derived. Its applicability to other models of incident radiation cannot be assumed. Indeed, its applicability within the same model to predict incident radiation at distances other than those at which $F$ was determined may also need to be considered carefully. For example, if $F$ was determined from experimental data of incident radiation in the far-field from a jet fire together with a particular model of the incident radiation field, and then the model applied to predict radiation in the near-field, erroneous results may arise due to the limitations of the modelling of the flame shape and size amongst other things. These issues are explored more fully in the next section.

\section{USING THE POINT SOURCE MODEL}

The single point source model (hereafter SP model) assumes that the flame shape is not important when calculating the incident radiation received outside the flame; an assumption which is clearly invalid near the flame. However, in the far-field this model can be very successful in predicting the incident radiation and is widely used. For the SP model, the flame is represented by a single point, usually located half-way along the flame axis, and incident radiation $\left(q_{S P S}\right)$ at a distance $\mathrm{S}$ (see Fig. 1$)$ determined as:

$q^{S P S}=\frac{F m H \tau_{S}}{4 \pi S^{2}}$

where $q^{S P S}$ is the incident radiation at distance $S\left(\mathrm{~kW} \mathrm{~m}^{-2}\right)$ onto a flat surface aligned to face the point source, (the superscript SPS denoting that the incident radiation is evaluated on a Single Point source basis with the distance S); $m$ is the mass flowrate $\left(\mathrm{kg} \mathrm{s}^{-1}\right)$; $H$ is the 
net calorific value of the fuel $\left(\mathrm{kJ} \mathrm{kg}^{-1}\right)$; $\tau_{S}$ is the atmospheric transmissivity evaluated over a distance $S$ with the prevailing atmospheric conditions (temperature and humidity); $S$ is the distance between the single point source located at the midpoint of the flame and the receiver (m), as shown on Fig.1. More generally, if the receiver is a flat surface, such that the angle between the normal to this surface and the line of sight to the point source in the centre of the flame is $\varphi$ as shown on Fig. 2, then:

$q^{S P S}=\frac{F m H \tau_{S}}{4 \pi S^{2}} \cos \varphi$

The SP model of incident radiation has been widely used, together with actual measurements of incident radiation $\left(q^{\text {meas }}\right)$, in order to determine the fraction of heat radiated when this is unknown for a particular fuel/release condition. In this case, by rearranging Eq. (6), the fraction of heat radiated, $F_{S P S}$, is given by:

$F_{S P S}=\frac{q^{m e a s} 4 \pi S^{2}}{m H \tau_{S}}$

EN ISO 23251 [11], Cook et al [12] and Lowesmith et al [3] used Eq. (8) to derive values of $F_{S P S}$ from experimental data, whilst others (Turns and Myhr [7]; Houf and Schefer, [8]; Oenbring and Sifferman, [13]; Schefer et al [14]) use Eq. (8), without the transmissivity term. In which case:

$q^{S P S}=\frac{F m H}{4 \pi S^{2}}$

and

$F_{S P S}=\frac{q^{\text {meas }} 4 \pi S^{2}}{m H}$ 
The omission of the transmissivity term could lead to significant differences when the distance, $S$, is large, since atmospheric absorption will reduce the radiation received, as can be seen in Fig. 3, which shows a correlation for atmospheric transmissivity developed from the data of Kondratyev, [15] over a range of distances and for flame temperatures of 1200 $\mathrm{K}, 1500 \mathrm{~K}$ and $2000 \mathrm{~K}$ which cover the range of temperatures typical of those found in large scale jet fires. The correlation also includes atmospheric temperature and relative humidity. At small distances (say less than 3m), typical of those which might be used during laboratory experiments on jet fires, the transmissivity $\sim 1$, so the lack of the term in Eqs. (9) and (10) is not significant, and hence its omission should not adversely affect the derivation of $F$ using the SP model and such small scale experimental data. However, if the model is then applied to large scale jet fires, to predict incident radiation at large distances, the inclusion of the transmissivity term is important. This appears to have been neglected by Houf and Schefer [8], who applied this approach to large scale hydrogen jet fires to predict the distance to given radiation levels.

Apart from the omission of the transmissivity, some of the above workers also made measurements of incident radiation based on the distance $\mathrm{R}$ rather than $\mathrm{S}$ as shown on Fig. 1 , although when the receiver was located half way along the flame axis, $R=S$, (which was often the case). However, there is another problem: incident radiation determined by the point source approach is known to be inaccurate in the near-field, so using this model together with experimentally determined values of incident radiation made in the nearfield, might not yield a useful value for $F$, as it is likely to appear to be dependent on the 
location at which it was determined. Conversely, if a true value of $F$ is known, this model will not predict accurately in the near-field, especially if the distance used is $R$ rather than $S$ and the receiver is located at an axial position towards the flame extremities. This issue was explored by Sivathanu and Gore [16], who made measurements of incident radiation at various distances along the flame axis, and at a radial distance of $R=0.5 L_{f}$, where $L_{f}$ is the flame length (m), as shown on Fig. 4. A 'true' value of $F$ was determined by numerically evaluating the radiative heat transfer equation to determine $Q_{r}$ and hence $F$ by Eq. (1). Then comparisons were made between the measured values of incident radiation at the various axial positions and that predicted by the SP model using $R$ as the distance and not including the transmissivity term, that is:

$q^{S P R}=\frac{F m H}{4 \pi R^{2}}$

The value of $F$ used in this calculations was the 'true' fraction of heat radiated determined as described above. Sivathanu and Gore [16], found that if they normalised the measurements of incident radiation, $q^{\text {meas }}$, at an axial location $z$ (along the flame axis expressed as a fraction of the flame length) by dividing by $q^{S P R}$, then the data collapsed onto a single curve. The bounds of the data are shown on Fig. 5. They termed this normalised incident radiation $C^{*}$ given by:

$$
C^{*}=\frac{q^{\text {meas }}}{q^{S P R}}
$$

and observed that it peaked at approximately 0.85 at axial locations between about $0.5 L_{f}$ to $0.7 L_{f}$. What this implies is that the true incident radiation, at a radial distance of $0.5 L_{f}$ and approximately halfway along the flame axis, is about 0.85 times that which would be 
calculated by Eq. (11). Sivathanu and Gore [16] conclude that, using this approach, measurements of incident radiation at an axial distance of about $0.5 L_{f}$ together with a value of $C^{*}$ of between 0.85 and 0.9 can be used to determine the fraction of heat radiated from a fire where this is unknown, using:

$F=\frac{q^{m e a s} 4 \pi R^{2}}{m H C^{*}}$

where $q^{\text {meas }}$ is the measured incident radiation at an axial distance of $0.5 L_{f}$. This conclusion is made regardless of the radial distance at which the incident radiation was made, despite their own observation that from theoretical considerations, $C^{*}$ must approach unity for large radial distances. However, as will be seen from the analysis below, there is even more concern if the measurement of incident radiation, $q^{\text {meas }}$, was determined at a radial position closer than $0.5 L_{f}$ or was made at an axial location where $C^{*}$ is not at its maximum value. Strictly speaking, Eq. (13) can only be used when $q^{\text {meas }}$ was determined at a radial location of $0.5 L_{f}$ (since this is the only value for which Sivathanu and Gore [16] present data) and then taking the appropriate value $C^{*}$ for the axial location where the measurement was made, rather than assuming it to be 0.85 . Furthermore, transmissivity should be included if the distances involved are not small.

\section{MODELLING NEAR-FIELD AND FAR-FIELD RADIATION}

Sivathanu and Gore [16] also showed that predictions from a weighted multi-ray calculation of radiative heat transfer could replicate the data shown in Fig. 5, within the bounds of its scatter, demonstrating the validity of their model even in this near-field 
region. For this current work, a similar modelling approach is adopted to first show that the data of Fig. 5 can be predicted satisfactorily, showing that the model can predict nearfield as well as far-field radiation. (The main advantage of deriving $C^{*}$ for this work is that it enables models to be compared with each other and with experimental data in a consistent manner). Then using this model, the sensitivity of $C^{*}$ is explored further by predicting its profile at other radial distances. Use of other large scale data provides further validation of the modelling approach taken in both the near-field and far-field. For convenience, and to facilitate use of further superscripts, the term $C^{*}$ shall hereafter be termed generically as $C$. For receivers orientated such that the normal to the surface of the receiver is perpendicular to the flame axis, (as shown on Fig. 4), $C_{x}$ shall be used, and for receivers whose normal is parallel to the flame axis, $C_{z}$ shall be used.

The model adopted here is a weighted multi-point source (WMP) model, whereby the radiation emanates from a number of point sources distributed along the flame axis, and the received incident radiation is determined as the vector sum of the radiation from each individual point source. For this model, the length of the flame must be predicted in order to locate the point sources, and the correlation of Lowesmith et al [3] can be used which is derived from extensive large scale data, that is:

$L_{f}=2.8893 Q^{0.3728}$

Provided at least 20 point sources are used, the incident radiation is independent of the number of point sources. However, the point sources were also weighted such that: 


$$
\begin{array}{ll}
w_{j}=j w_{1} & \text { for } j=1, \ldots, n \\
w_{j}=\left[n-\frac{(n-1)}{(N-(n+1))} \cdot(j-(n+1))\right] w_{1} & \text { for } j=n+1, \ldots, N \\
\sum_{j=1}^{N} w_{j}=1 &
\end{array}
$$

where $1 \leq n \leq N$, the number of point sources. In this work, $n=0.75 N$. (The equations for the weighting result in the weighting increasingly linearly to the $n$th point (where $w_{n}=n w_{1}$ and then decreasing linearly from the $(n+1)$ th point where $w_{n+1}=w_{n}$ such that $w_{N}=w_{1}$ ). This weighting of the point sources was based, to a certain extent, on measurements made on large scale jet fires using a narrow angle radiometer reported by Cook et al [12] together with the observation that the radiation tends to increase to a peak about $3 / 4$ of the way along the flame. A linear variation in the weighting on either side of the peak was assumed for simplicity, although more complex variations could have been attempted. The location of the peak also agrees reasonably with the data of Sivathanu and Gore [16] and Baillie et al [18]. It should be noted that the variation in weighting of the point sources along the flame trajectory and position of the peak used in this paper are not intended to be final solutions, they were merely used to illustrate the potential of the multipoint source approach.

Incident radiation at a location, outside the fire, as shown on Fig. 6, is given by the vector sum: 
$q^{W M P}=\sum_{j=1}^{N} \overrightarrow{q_{J}}=\sum_{j=1}^{N} \frac{w_{j} F m H \tau_{j}}{4 \pi \overrightarrow{S_{J}^{2}}} \cdot \cos \varphi_{j}$

where $w_{j}$ is the weighting of the $j$ th point source given by Eq. (15); $\tau_{j}$ is the transmissivity over the distance $S_{j}$, from the $j$ th point source to the receiver and $\varphi_{j}$ is the angle between the normal of the receiver and the line of sight to the $j$ th point source.

In the far-field, this model approximates to the SP model and can give reliable predictions of incident thermal radiation (within $\pm 20 \%$ ). This is illustrated on Fig. 7 which presents data from a large scale jet fire both within and beyond one flame length from the fire. However, unlike the SP model, the WMP model can also provide reliable predictions in the near-field. This can be illustrated by predicting values of $C_{x}=\frac{q^{W M P}}{q^{S P R}}$ at a radial distance of $0.5 L_{f}$ and varying axial location, and then comparing with the data for $C_{x}$ given by Sivathanu and Gore [16] as shown on Fig. 8. (This is termed $C_{x}^{W M P / S P R}$ so that the superscript provides information on the model used and the basis of comparison, that is, the SP model based on $R$ ). As can be seen, the predicted values of $C_{x}^{W M P / S P R}$ peak in the correct region in the axial direction and the curve lies within the bounds of the data presented by Sivathanu and Gore [16]. This provides confidence in the validity of the modelling approach in the near-field region. Furthermore, $C_{x}^{W M P / S P R}$ can now be predicted for different radial distances. Figures 9 (a) and (b) provide further validation of the modelling approach in the near-field by comparing with some confidential data (GL Noble Denton [17]) used previously by Lowesmith et al [3] and data from Baillie et al [18] with 
predictions at the relevant locations. Figure 9 (a) shows $C_{x}^{W M P / S P R}$ predictions compared with the data at radial distances of $0.35 L_{f}, 0.7 L_{f}$ and $0.9 L_{f}$ and demonstrates reasonable agreement. Figure 9 (b) shows $C_{z}^{W M P / S P R}$ for receivers with their normal parallel to the flame axis and located at $\mathrm{z}=0$, for varying radial distances $(\mathrm{x})$. As can be seen, the trend is good, but with some slight over-prediction. It should also be borne in mind that in reality the flame envelope itself would very likely extend to a diameter of $0.17 L_{f}$ (Sivathanu and Gore [16]), so predictions of incident radiation very close are not meaningful.

(It is worthy of note here that an advantage of using values of $C$ to compare models with each other and with data is that $C$ is insensitive to atmospheric transmissivity since it appears both in the numerator and denominator and the ratio is almost always approximately unity. However, when a chosen model is then used to predict incident radiation, it is important to evaluate and include atmospheric transmissivity especially for predictions at significant distances from a fire).

Having established the ability of the WMP model to predict both far and near-field radiation, Fig. 10 presents predicted $C_{x}^{W M P / S P R}$ from $0.25 L_{f}$ to $5 L_{f}$. As expected, for larger radial distances, the peak is close to 1 and is much flatter, showing that in the farfield the model approximates to a point source. However, as can be seen, as the radial distance decreases, the peak occurs at a lower value and the variation with axial distance is much more intense. This highlights the danger in the recommendation of Sivathanu and 
Gore [16] to use a value of 0.85 to 0.9 for $C_{x}$ regardless of the radial distance, when using measurements of incident radiation to determine the fraction of heat radiated by Eq. (13). As can be seen on Fig. 10, the value of $C_{x}$ can be significantly less than 0.8 if the radial location is less than $0.5 L_{f}$ and varies strongly with even small changes in axial location, giving the potential for large errors.

Note that Fig. 10 is presenting $C_{x}^{W M P / S P R}$, which is the normalised incident radiation for a receiver whose normal is perpendicular to the flame axis. In many cases, when radiation measurements are made of fires, the radiometers are actually aligned such that they receive the maximum radiation for that location, that is, towards the central region of the fire, this would produce $C_{\max }$ when normalised by dividing by $q^{S P R}$. At an axial location of around $0.5 L_{f}$ to $0.7 L_{f}, C_{\max }$ is approximately the same as $C_{x}$ and Fig. 11 shows $C_{\max }^{W M P / S P R}$ as it varies with radial distance. What this demonstrates is that, when the receiver is located about halfway along the flame axis, for radial distances over about one flame length $\left(L_{f}\right)$, the difference between the point source approach (based on $R$ ) and the correct incident radiation is less about $5 \%$ (since $C_{\max }^{W M P / S P R} \sim 0.95$ ), giving an indication of the extent of the 'near-field', (within which SP model calculations (using $R$ ) of incident radiation would need to be adjusted). However, also shown on Fig. 11 is the predicted $C_{\max }^{W M P P R}$ for a receiver at an axial location at the beginning of the flame $(z=0)$ as it varies with radial distance. This is typical of the situation likely to arise when experiments to study large scale vertical jet fires are undertaken with receivers positioned at ground level, orientated 
towards the central region of the flame. As can be seen, a larger radial distance is required before $C_{\max } \sim 1$, so for this arrangement, the 'near-field', where SP model calculations (based on R) will be inaccurate, extends to a greater radial distance.

\section{EXPLORING NEAR-FIELD BEHAVIOUR OF VARIOUS MODELLING APPROACHES}

In the previous section, it was shown that a weighted multi-point source model could predict near-field behaviour by demonstrating that it could replicate the data of Sivathanu and Gore [16] for $C_{x}$ and Baillie et al [18] for $C_{x}$ and $C_{z}$. Different modelling approaches can also be considered and by comparing with the data, the inherent characteristics of the model can be explored in terms of: (a) the likely potential of the model to be able to predict near-field incident radiation and; (b) how the fraction of heat radiated to be used for the model should be adjusted compared to the 'real' value $(F)$, which can also be taken to be that derived from the WMP model, $F_{W M P}$.

Firstly, the SP model (based on the distance $S$ between the point source and the receiver) is considered (termed SPS), and then various solid flame geometries. 


\subsection{Single Point Source Model (SPS)}

Referring to Figs. 2 and 4, for a receiver inclined such that its normal is perpendicular to the flame axis, $\varphi=\theta$, hence:

$q_{x}^{S P S}=\frac{F m H \tau_{S} \operatorname{Cos} \theta}{4 \pi S^{2}}$

So, $C_{x}^{S P S / S P R}=\frac{q_{x}^{S P S}}{q_{x}^{S P R}}=\frac{F m H \tau_{S} \operatorname{Cos} \theta}{4 \pi S^{2}} \cdot \frac{4 \pi R^{2}}{F m H \tau_{R}}=\frac{R^{2} \cos \theta \tau_{S}}{S^{2} \tau_{R}}$

Assuming that the ratio of transmissivities is approximately 1 , and since $R=S \operatorname{Cos} \theta$, then:

$C_{x}^{S P S / S P R} \sim \operatorname{Cos}^{3} \theta$

It can also be noted that for receivers aligned such that their normal is parallel to the flame axis (perpendicular the radial direction), then it can be shown that:

$C_{z}^{S P S / S P R} \sim \operatorname{Cos}^{2} \theta \operatorname{Sin} \theta$

and for receivers aligned to receive the maximum radiation, $\varphi=0, \operatorname{Cos} \varphi=1$, so,

$C_{\max }^{S P S / S P R} \sim \operatorname{Cos}^{2} \theta$

which is also $\sqrt{\left(C_{x}^{S P S / S P R}\right)^{2}+\left(C_{z}^{S P S / S P R}\right)^{2}}$.

$C_{x}^{S P S / S P R}$ is shown on Fig. 12 for a radial distances of $0.35 L_{f}$ and $0.5 L_{f}$ for varying positions along the flame axis, $z$. As can be seen, the agreement with the data is poor, in terms of both the shape of the curve and the location and magnitude of the peak. What this is saying is that the SPS model is inherently unsuitable in the near-field. This can also be 
demonstrated by comparing incident radiation from the SPS model to that from the WMP model (which has been shown to agree with data in the near-field). That is, defining;

$C_{x}^{S P S / W M P}=\frac{q_{x}^{S P S}}{q_{x}^{W M P}}=\frac{q_{x}^{S P S}}{q_{x}^{S P R}} \cdot \frac{q_{x}^{S P R}}{q_{x}^{W M P}}=\frac{C_{x}^{S P S / S P R}}{C_{x}^{W M P / S P R}}$

This is shown on Fig. 13, for a range of radial distances. The deviation from 1, particularly over the length of the flame for short radial distances, indicates the limitations of the SPS model.

Further, if experimental data taken in the near-field is used in the SPS model to derive a fraction of heat radiated directly, then this value will need correction to enable its use more generally. (This is also the case if the SP model is used with the distance $R$, termed SPR). For example, assuming measurements are taken for receivers aligned with their normal perpendicular to the flame axis, and based on the distance $\mathrm{R}$ then:

$F_{S P R}=\frac{q_{x}^{\text {meas }} 4 \pi R^{2}}{m H \tau_{R}}$

But from Eq. (12), since the WMP model agrees with the data of Fig. 5;

$C_{x}^{W M P / S P R}=\frac{q_{x}^{W M P}}{q_{x}^{S P R}}=\frac{q_{x}^{\text {meas }}}{q_{x}^{S P R}}=\frac{q_{x}^{\text {meas }} 4 \pi R^{2}}{F m H \tau_{R}}$

Hence, using Eq. (23), the true fraction of heat radiated, $F$, is given by:

$$
F=\frac{q_{x}^{\text {meas }} 4 \pi R^{2}}{m H \tau_{R} C_{x}^{W M P / S P R}}=\frac{F_{S P R}}{C_{x}^{W M P / S P R}}
$$


This approach was adopted by Studer et al [10] to determine the fraction of heat radiated from hydrogen flames from experimental measurements taken by radiometers aligned with their normal perpendicular to the flame axis and approximately half-way along the flame axis and $0.5 L_{f}$ radial distance.

Similarly, if the measurements are based on the distance S, it can be shown that:

$F=\frac{F_{S P S} \operatorname{Cos}^{3} \theta}{C_{x}^{W M P} / S P R}$

Or, if the receiver is aligned to receive the maximum incident radiation,

$F=\frac{F_{S P S} \operatorname{Cos}^{2} \theta}{C_{x}^{W M P} / S P R}$

Of course, the appropriate value of $C_{x}^{W M P / S P R}$ must be used according to the axial and radial location, which can be obtained from Fig. 10, and care taken if this is in a region where $C_{x}^{W M P / S P R}$ is varying strongly. In the far-field, $\operatorname{Cos} \theta \sim 1$ and $C_{x}^{W M P / S P R} \sim 1$, so from Eq. (25) $F=F_{S P R}$ and from Eq. (26) $F=F_{S P S}$. Lowesmith et al [3] evaluated the fraction of heat radiated from an extensive body of large scale jet fire data, based on the single point source model using the distance $S$ and including the transmissivity. Hence the fraction of heat radiated derived was $F_{S P S}$. For natural gas jet fires, the average value was 0.13. The data for the natural gas jet fires has since be re-evaluated using the WMP model and no significant change was observed $(<10 \%)$, since the authors were already aware of the need to use far-field measurements and only radiometers more than 1 flame length away were used in the derivation of $F_{S P S}$. (However, the opportunity was also taken to 
include some data from Cook et al [12] in the overall average for fraction of heat radiated, which had not been included previously, and the overall average value, for high pressure (sonic) natural gas releases was found to be $F=0.15 \pm 0.04$.

Finally, it is important to emphasise again, that if $F$ has been derived from essentially laboratory scale data and the transmissivity term taken to be unity, then, when predictions of large scale jet fires are undertaken at larger distances, it is important to include transmissivity in the calculation of incident radiation. It appears that Houf and Schefer [8], in calculating incident radiation for a range of jet fires (some large scale) and a range of radial distances did not include transmissivity. Furthermore, as the calculations were performed using the SP model and the distance $\mathrm{R}$, the true incident radiation is actually $q_{x}^{S P R} . C_{x}^{W M P / S P R}$, where $C_{x}^{W M P / S P R}$ is given by Fig.10 at the appropriate axial and radial location. However, it appears that Houf and Schefer [8] took the erroneous advice of Sivathanu and Gore [16] to take $C^{*}=C_{x}^{W M P / S P R}$ as a constant value of 0.85 to 0.9 for all radial distances. This will inevitably lead to errors in the calculation of incident radiation, especially in cases where the radial distances were as little as $0.2 L_{f}$.

\subsection{Solid Flame Models}

A common approach to modelling of jet fires is to assume the fire corresponds to an idealised shape, such as a cylinder or cone. If a solid shape is adopted, then the incident radiation from the fire is given by: 
$q=V E \tau_{\text {sol }}$

where, $E$ is the surface emissive power $\left(\mathrm{kW} \mathrm{m}^{-2}\right)$ and $E=\frac{Q_{r}}{A}=\frac{F Q}{A}$. For the location and orientation of the receiver; $V$ is the view factor of the flame and $\tau_{\text {sol }}$ is the transmissivity based on the distance to the flame surface. To use models of this type, the dimensions of the flame must be determined and the value of $E$, either directly or via $F$. Note that since the idealised shape given to the flame may not have the same area as the real flame, the value of $E$ may differ from that measured for a real fire. Similarly, a value of $E$ established for a model using one flame shape, cannot be used in another model based on a different shape. However, what follows here may assist in the transfer of information derived for $F$ or $E$ from one model to another.

A computer programme was used to calculate the view factor of a chosen receiver location and orientation relative to the chosen flame shape (Hankinson [19]. Then incident radiation can be determined for a chosen location as:

$q^{s o l}=V E \tau=\frac{V F m H \tau_{s o l}}{A}$

and this can be normalised as described previously by dividing by $q^{S P R}$ to obtain $C_{x}^{S o l / S P R}$.

Thus, $C_{x}^{S o l / S P R}=\frac{V F m H \tau_{s o l}}{A} \cdot \frac{4 \pi R^{2}}{F m H \tau_{R}}=\frac{V 4 \pi R^{2}}{A}$

Since $\tau_{\text {sol }} \sim \tau_{R}$. It can be seen that the above analysis is independent of $F$ provided the same value is taken for the solid flame model as for the evaluation of $q^{S P R}$. The values of $C_{x}^{S o l / S P R}$ can then be compared with the data of Sivathanu and Gore [16] and the values of 
both $C_{x}^{\text {sol/SPR }}$ and $C_{z}^{\text {sol/SPR }}$ compared with the data of Baillie et al [18] in order to assess the near-field behaviour of this model, as was described previously for the SPS model. Similarly, it can be compared with the WMP model by deriving:

$$
C_{x}^{S o l / W M P}=\frac{C_{x}^{s o l / S P R}}{C_{x}^{W M P / S P R}}
$$

Some typical flame shapes are now considered, namely, a cylinder (CYL), a cone (CON), a back-to-back cone (BBC) and a back-to-back weighted ellipsoid (BBE) as shown diagrammatically on Fig. 14. The assumed dimensions for each shape are shown on Fig. 14. It was also assumed that the surface emissive power (or fraction of heat radiated) was uniform over the surface. As per Eq. (30), $C_{x}^{C Y L / S P R}, C_{x}^{C O N / S P R}, C_{x}^{B B C / S P R}$ and $C_{x}^{B B E / S P R}$ were calculated for the four shapes respectively. The variation of these values with axial distance are shown in Figs. 15 (a) to (d) and compared with the bounds of the near-field experimental data of Sivathanu and Gore [16] and Baillie et al [18]. From Fig. 15 (a) it can be seen that the cylindrical flame shape over-predicts the data close to the beginning of the fire. Furthermore the location and magnitude of the peak do not agree well with the data. Some improvement could be achieved by appropriate weighting of the emissive power at different locations along the flame length. Another way of weighting the radiant emissions is to vary the flame width at different axial locations, which is essentially accomplished by considering the cone compared to the cylinder. As can be seen in Fig. 15 (b), the conical flame shape shows slightly better agreement than the cylinder, but the peak still occurs in the wrong place and is high (above the band of data for Sivathanu and Gore [16], and well above the peak of the data from Baillie et al [18]). Some additional 
weighting of the emissive power might improve matters. Figure 15 (c) presents the results for the back-to-back cone which agrees well in terms of shape and location of the peak, but the predicted values are generally high compared to the data. The best agreement with the data, of the four flame shapes, is demonstrated in Fig. 15 (d) for the back-to-back ellipsoid, which peaks in the correct location, although slightly high, and is in reasonable agreement at both short and long axial positions.

Figures 16 (a) to (d) present $C_{z}^{\text {sol/SPR }}$ for the four flame models compared with the relevant data from Baillie et al [18]. At first glance, Fig. 16(a) appears to provide the best agreement. However, the predictions drop to zero at $x=0.0425 L_{f}$ since this location is at the flame surface of the cylinder where the view factor of a receiver whose normal is parallel to the $z$ axis is zero. Then as $x$ increases, the cylindrical model over-predicts initially, but it can be seen that for larger values of $x$, the predictions are tending towards under-prediction. For the other three models, Figs. 16(b) to (d) show under-prediction. This is an artefact of the solid flame shape viewfactor modelling approach, whereby the radiant power emitted by an element of the flame surface varies as the cosine of the angle to the normal of the element.

Figures 17 (a) to (d) present the comparison of the respective models against the WMP model by determining $C_{x}^{C Y L / W M P}, C_{x}^{C O N / W M P}, C_{x}^{B B C / W M P}$ and $C_{x}^{B B E / W M P}$. Here, the behaviour of the model over a range of radial distances and axial locations can be observed. 
By inspection of all four figures for large radial distances $\left(R=5 L_{f}\right)$, it is apparent that $C_{x}^{\text {sol/WMP }}$ is about 1.2, indicating that the far-field behaviour of these solid flame models is different to the WMP model and hence the true fraction of heat radiated, $F$, would need to be adjusted for use with all these solid flame shapes to predict far-field radiation by a factor of $\frac{1}{1.2}=0.83$. As indicated by the profiles at shorter radial distances, the correction factor to provide accurate predictions of incident radiation in the near-field by these solid flame shapes varies with position, in some cases considerably, highlighting the difficulty of modelling near-field radiation with these solid flame shapes. In addition, on Figs. 17 (a) and (b), at close radial distances, the profiles exhibit non-smooth behaviour with kinks appearing close to an axial position of one flame length. This arises due to different surfaces of the shape coming into view of the receiver as the position of the receiver changes (for example, the end face of the cylinder or cone appearing in the field of view of the receiver). What this means is that the contours of incident radiation around a fire predicted by these models do not vary smoothly. Figure 17 (a) also shows the same effect as Fig. 15 (a), in as much as in the near-field, the profiles are greater than 1 in the first part of the flame and less than 1 in the later stages of the flame. Similarly, in Fig. 17 (b), the near-field profiles are greater than 1 for axial locations more than about 0.6 of the flame length to the end of the flame. The back-to-back cone shown in Fig. 17 (c) is better behaved, in that no kinks are apparent in the profiles and they are more uniform along the length of the flame. Similar, but slightly improved behaviour, can be seen in Fig.17 (d) for the back-to-back weighted ellipsoid. 


\subsection{Distributed Point Source on Solid Flame Shape}

Finally, one further modelling approach has been explored, whereby the best solid flame shape (the back-to-back weighted ellipsoid) was selected and then hemispherical point sources distributed over its surface. Each point source represents a small portion of the flame area and the contribution of each point source to the total radiation is in proportion to the area of the flame that it represents as a fraction of the total flame area. The incident radiation is then determined by a vector sum of the radiation from each point source in the same way as for the multi-point source model, except that only point sources which can 'see and be seen by' the receiver are included in the summation. This distributed point source approach applied to the back-to-back weighted ellipsoid (DPE) can be evaluated in the same way as above for the solid flame models by determining $C_{x}^{D P E / S P R}$ and $C_{z}^{D P E / S P R}$ for comparison with available data, and by determining $C_{x}^{D P E / W M P}$. These are presented on Fig. 18 (a), (b) and (c) respectively. Comparison of Fig. 18 (a) with Figs. 8 and 9 (a) demonstrates that the predictions of the DPE model are very similar but improved compared with the performance of the WMP model, in the near-field region as the receiver moves parallel to the flame axis. Figure 18 (b) confirms this observation for a wide range of radial distances. Finally, Fig. 18(c) shows improved performance of the DPE model compared to the WMP model as shown on Fig. 9 (b), for receivers at the beginning of the flame, facing parallel to the flame axis. 


\section{DISCUSSION AND CONCLUSIONS}

This study has highlighted the dependence of the fraction of heat radiated on the method of its derivation from experimental data, using a model of the incident radiation field. It may be the case that the fraction of heat radiated generated by one model will not be applicable to another without some adjustment. Additionally, if incident radiation data in the nearfield is used to derive the fraction of heat radiated, then some correction may be needed to be applied to determine the 'true' fraction of heat radiated, that is, a value which is independent of the location around, and the distance from, a fire. The study has identified a method by which the validity and general applicability of a published value of fraction of heat radiated can be assessed in terms of its suitability to predict near-field and far-field radiation.

Although it would always be preferable to derive the fraction of heat radiated from incident radiation data using the same model as is intended to be used subsequently, this is not always possible as the detailed experimental data is not always published. For such situations, this study provides a mechanism by which the fraction of heat radiated generated by one method, can be adjusted so that it would be suitable for another model of incident radiation. This greatly assists the use of published data on fraction of heat radiated, provided the basis of its derivation is stated. 
The method of Sivathanu and Gore [16], by which a single near-field measurement of incident radiation is used to facilitate derivation of a true fraction of heat radiated for a flame by determining the fraction of heat radiated based on a single point source model and then including a correction factor, has been explored in some detail. This has shown that the correction factor varies with the radial distance from the jet fire and may be significantly lower than the 0.85 to 0.9 suggested by Sivathanu and Gore, for radial distances less than half the flame length. The correction factor also varies strongly with axial position of the receiver, especially at near-field positions, which potentially leads to significant error in identifying the appropriate correction factor to apply. Whilst the method may be useful for the interpretation of laboratory scale data, where the flames and receiver positions can be closely controlled, it is likely to lead to significant error if applied to large scale data undertaken outdoors due to variability of conditions in such environments. This suggests that it is preferable to use far-field measurements of incident radiation in order to derive the fraction of heat radiated wherever possible.

The single point source method has been used by several workers, to derive fraction of heat radiated (which is quite satisfactory if far field data is used) and, in some cases, also to predict incident radiation fields from real fires. However, some workers have not included atmospheric transmissivity in the model, which can be significant when considering large scale jet fires, where the distance to the receiver of interest may be large. 
The correction factors identified by Sivathanu and Gore [16] in the near-field, have been extended herein to provide a means by which modelling approaches for incident radiation around a jet fire can be assessed in terms of their inherent characteristics to be able to predict near-field and far-field radiation. By assessing the inherent characteristics of the model, it provides a basis by which adjustments can be explored, such as modifying the shape of the flame or weighting the contribution from different regions of the flame. Taking this approach, it was shown that the single point source model (with a single point located at half the flame length) was inherently unsuitable to predict near-field radiation as it provided a poor match to data. However, a weighted multi-point source approach was shown to be able to predict both far and near-field radiation, enabling this to be used as a method to determine the 'true' fraction of heat radiated by a flame from experimental data and providing a measure against which other modelling approaches can be assessed.

Four idealised solid flame shapes were considered: a cylinder, a cone, a back-to-back cone and a back-to-back ellipsoid. Their near-field behaviour identified that some weighting of the fraction of heat radiated (or surface emissive power) would be preferable to improve their ability to provide predictions in the near-field. By examining their behaviour in the near-field and far-field relative to the weighted multi-point source model, it is also possible to determine how to adjust the true fraction of heat radiated (and hence surface emissive power) to enable its use with a particular flame shape. The far-field behaviour identified that the fraction of heat radiated for these solid flame shapes would be a factor 0.83 times that used for the multi-point source model. Due to the abrupt change in profile of the flame 
surface of the first two of these flame shapes, some non-smooth behaviour arises in the predicted incident radiation field as the receiver moves to certain axial positions, as a result of different faces of the flame coming into view. This suggests that a preferable flame shape would be one that provides a continuous smooth surface, more representative of a flame. A smooth flame shape, based on elliptical functions (back-to-back weighted ellipsoid), was studied and this shape demonstrated good potential for predicting both nearfield and far-field radiation with no discontinuities in the incident radiation field.

Finally, an approach in which a flame shape is combined with point sources was considered, by distributing hemispherical point sources over the surface of the solid flame shape. This approach was found to perform well in both the near-field and far-field. Furthermore, it was found that the true fraction of heat radiated needed no adjustment for use with this model and can be assumed constant throughout the near and far field and for any position or orientation of a receiver around the fire. This demonstrates the superior suitability of this modelling approach compared to any of the view factor models. Indeed, this modelling approach is perhaps more representative of the emission of radiation from a real flame, whereby the radiant power emitted by an element of the flame surface is uniform over the hemisphere subtending the point source, rather than varying as the cosine of the angle to the normal of the element, as is the case in the view factor modelling approach. 


\section{NOMENCLATURE}

$a_{f} \quad$ Planck mean absorption coefficient $\left(\mathrm{m}^{-1}\right)$

$C^{*} \quad$ (Also, generically, $\left.C\right)$. Normalised incident radiation $\left(=q^{\text {meas }} / q^{S P R}\right)$ as given by [16] for measured values. For predicted values of $C, q^{\text {meas }}$ is replaced by a predicted incident radiation using a selected model, eg $q^{W M P}$. (See below for more about model acronyms and sub and superscripts for C).

$d_{j} \quad$ Jet exit diameter (m)

$F \quad$ Fraction of heat radiated $\left(=Q_{r} / Q\right)$. No subscript means it is the 'true' value. Subscript indicates model used for determination.

$H \quad$ Net calorific value of the fuel $\left(\mathrm{kJ} \mathrm{kg}^{-1}\right)$

$L_{f} \quad$ Flame length (m)

$m \quad$ Mass flowrate $\left(\mathrm{kg} \mathrm{s}^{-1}\right)$

$M \quad$ Molecular weight $\left(\mathrm{kg} \mathrm{kmol}^{-1}\right)$

$N \quad$ Number of point sources for WMP model

$p_{j} \quad$ Absolute pressure at jet exit $\left(\mathrm{kg} \mathrm{m}^{-1} \mathrm{~s}^{-2}\right)$

$p_{\infty} \quad$ Absolute atmospheric pressure $\left(\mathrm{kg} \mathrm{m}^{-1} \mathrm{~s}^{-2}\right)$

$q$ Incident radiation $\left(\mathrm{kW} \mathrm{m}^{-2}\right)$. Subscript indicates alignment of receiver. Superscript meas indicates measured value. Other superscripts indicate model used to calculate incident radiation (see below for model acronyms and more about sub and superscripts for $q$.) 
Q Energy of combustion (kW)

$Q_{r} \quad$ Proportion of energy of combustion released as radiant energy (kW)

$R \quad$ Distance perpendicular to flame axis (m). See Figure 1.

$R_{u} \quad$ Universal gas constant $\left(\mathrm{J} \mathrm{kmol}^{-1} \mathrm{~K}^{-1}\right)$

$S \quad$ Distance (m). See Figure 1.

$t_{f} \quad$ Flame residence time (s)

$T_{a d} \quad$ Adiabatic flame temperature (K)

$u_{e f f} \quad$ Effective jet velocity $\left(\mathrm{m} \mathrm{s}^{-1}\right)\left(=u_{j}+\left(p_{j}-p_{\infty}\right) /\left(\rho_{j} u_{j}\right)\right.$ from [6])

$u_{j} \quad$ Jet exit velocity $\left(\mathrm{m} \mathrm{s}^{-1}\right)$

$w_{i} \quad$ Weighting of the ith point source in the WMP model.

$W_{f} \quad$ Flame width (m)

$x \quad$ Direction perpendicular to flame axis (radial).

$y_{s} \quad$ Mass fraction of fuel at stoichiometric conditions

Z Direction of the flame axis

\section{Greek Letters}

$\rho_{f} \quad$ Flame density $\left(\mathrm{kg} \mathrm{m}^{-3}\right)\left(=p_{\infty} M /\left(R_{u} T_{a d}\right)\right.$ from [8])

$\rho_{j} \quad$ Jet exit density $\left(\mathrm{kg} \mathrm{m}^{-3}\right)$

$\theta \quad$ Angle as shown on Fig. 2. 
$\varphi \quad$ Angle as shown on Fig. 2.

$\tau \quad$ Atmospheric transmissivity. Subscript indicates distance over which transmissivty is determined.

\section{Model Acronyms}

BBC Back-to-Back Cone model

BBE Back-to-Back weighted Ellipsoid model

CON Cone model

CYL Cylinder model

DPE Distributed Point sources on back-to-back Ellipsoid model

sol Any of the solid flame models

SPR Single Point Source Model using distance R

SPS Single Point Source Model using distance S

WMP Weighted Multi-Point Source model

\section{Sub and Superscripts for $C$ and $q$}

$x \quad$ Subscript for $C$ or $q$ determined for receivers such that the normal to the surface of the receiver is perpendicular to the flame axis (see Fig. 4).

z Subscript for $C$ or $q$ determined for receivers whose normal is parallel to the flame axis. 
$\max$ Subscript for $C$ or $q$ determined for receivers aligned to receive the maximum radiation

$A B C \quad$ Superscript for $q$ indicating model used to predict incident radiation. See Model Acronyms for values of $A B C$.

$A B C / S P R \quad$ Superscript for $C$ indicating predicted value of $C$ based on model 'ABC', normalised by model SPR, that is, $C^{A B C / S P R}=q^{\mathrm{ABC}} / q^{S P R}$.

ABC/WMP Superscript for $C$ indicating predicted value of $C$ based on model 'ABC', normalised by model WMP, that is, $C^{A B C / W M P}=q^{A B C} / q^{W M P}$.

meas Superscript for $q$ indicating it is a measured value.

\section{REFERENCES}

[1] G.R. Kent, Hydrocarbon Processing 43 (1964) 121-125.

[2] S.H. Tan, Hydrocarbon Processing 46 (1967) 172-176.

[3] B.J. Lowesmith, G. Hankinson, M.R. Acton, G. Chamberlain, Trans IChemE, Part B 85 (1987) 207-220.

[4] D.K. Cook, M. Fairweather, J. Hammonds, D.J. Hughes, Chem. Eng. Res. Des. 65 (1987) 318-325. 
[5] G.A. Chamberlain, Chem. Eng., Res. Des. 65 (1987) 299-309.

[6] A.D. Birch, D.R. Brown, M.G. Dodson, F. Swaffield, Combust. Sci. Technol. 36 (1984) 249-261.

[7] S.R. Turns, F.H. Myhr, Combust. Flame 87 (1991) 319-335.

[8] W. Houf , R. Schefer, R, Int. J of Hydrogen Energy 32 (2007) 136-151.

[9] A. Molina, R.W. Schefer, W.G. Houf, Proc. Combust. Inst. 31 (2006) 2565-73.

[10] E. Studer, D. Jamois, S. Jallais, G. Leroy, J. Hebrard, V. Blanchetiere, Int. J Hydrogen Energy 34 (2009) 9611-9619.

[11] EN ISO 23251, Petroleum, petrochemical and natural gas industries - Pressure relieving and depressurising systems, 2007. 
[12] D.K. Cook, M. Fairweather, J. Hammonds, D.J. Hughes, Chem. Eng. Res. Des. 65 (1987) 310-317.

[13] O.R. Oenbring, T.R. Sifferman, Proc. of the American Pet. Inst. Refining Dept. Published by API, 59 (1980) 220-236.

[14] R.W. Schefer, W.G. Houf, T.C. Williams, B. Bourne, J. Colton, Int. J Hydrogen Energy 32 (2007) 2081-2093.

[15] K.Y. Kondratyev, Radiative heat exchange in the atmosphere, Pergamon Press, New York, 1965.

[16] Y.R. Sivathanu, J.P. Gore, Combust. Flame 94 (1993) 265-270.

[17] GL Noble Denton, The effectiveness of water area deluge in mitigating the effect of fires, unpublished report, 1997.

[18] S. Baillie, M. Caulfield, D.K. Cook, P. Docherty, Trans. IChem E, Part B 76 (1998) 313. 
[19] G. Hankinson, Fire safety science, $1^{\text {st }}$ Int. Symp. Proc. NIST, USA, (1985). 


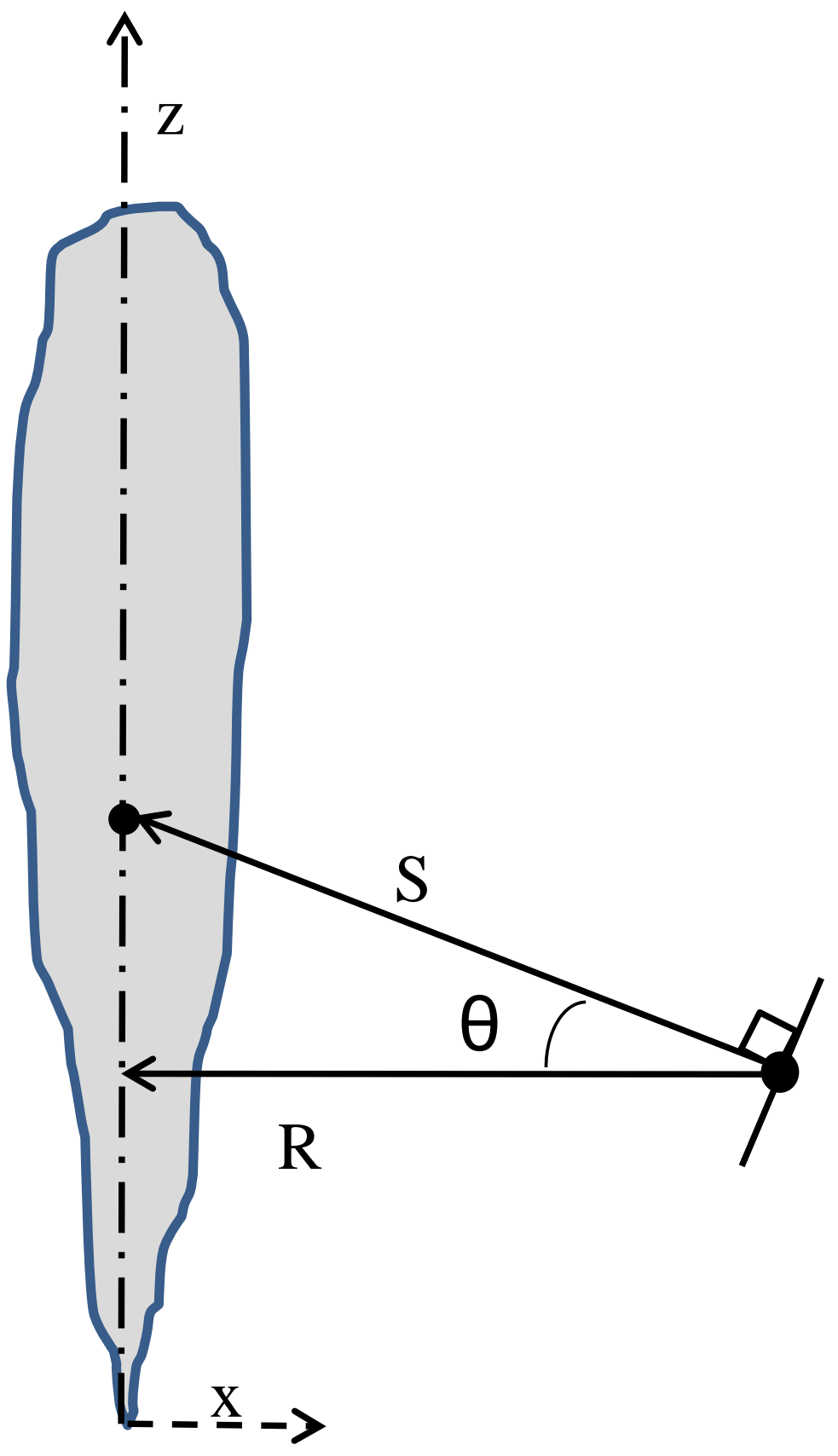

Fig.1: Single Point Source Model 


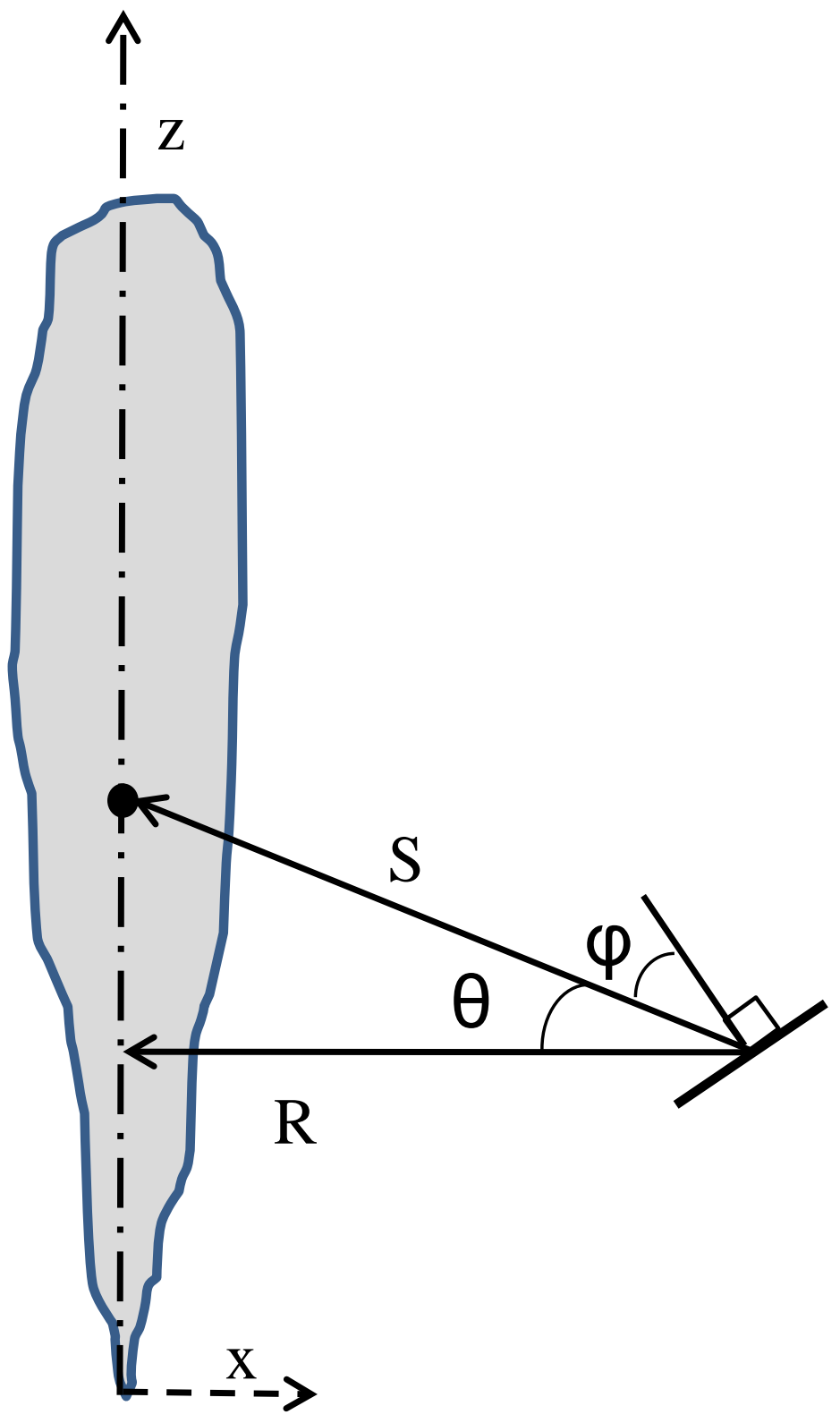

Fig.2: Single Point Source Model with inclined reciever 


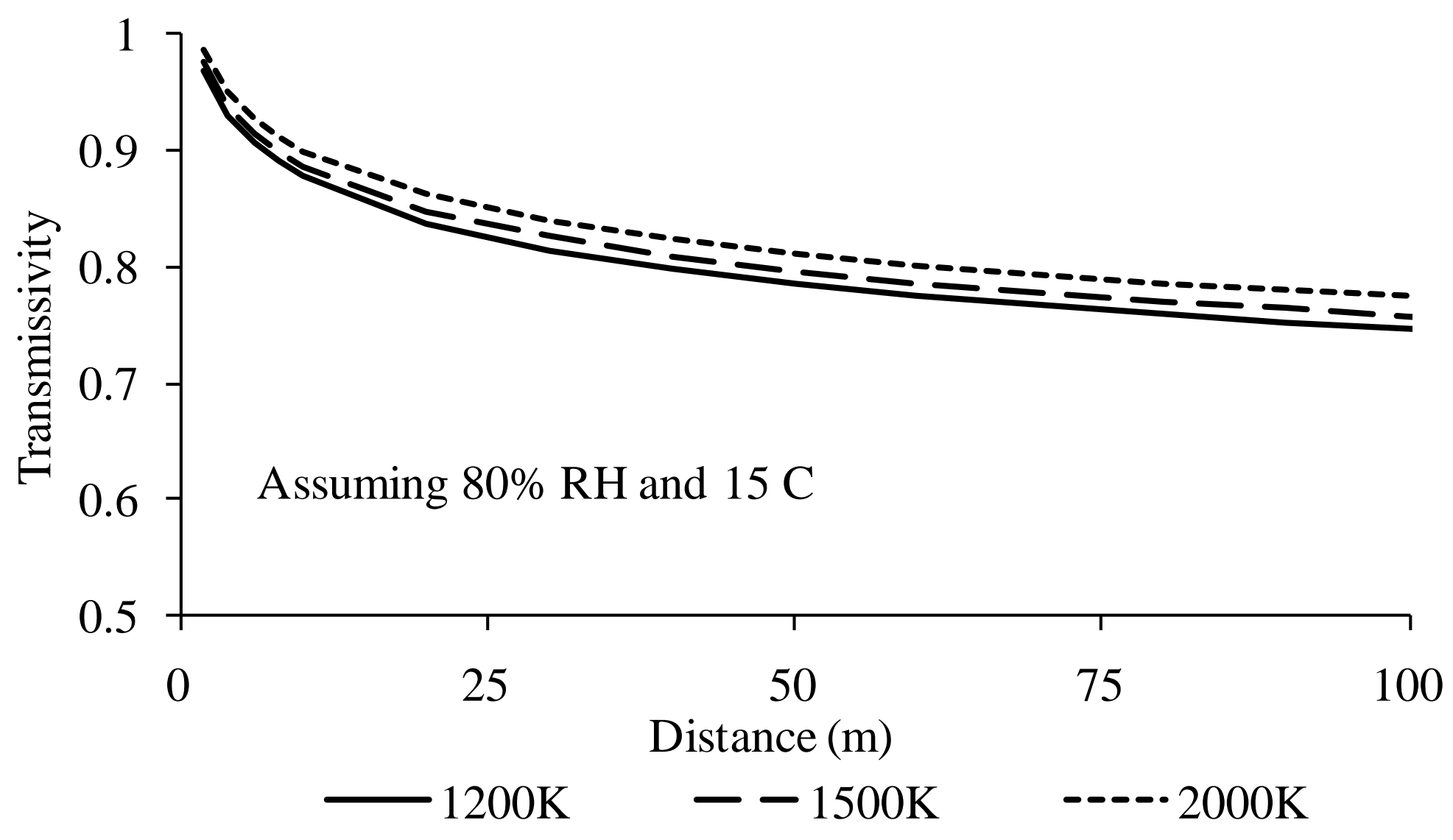

Fig. 3: Atmospheric transmissivity as a function of distance for three different flame temperatures, based on the data of Kondratyev [15] 


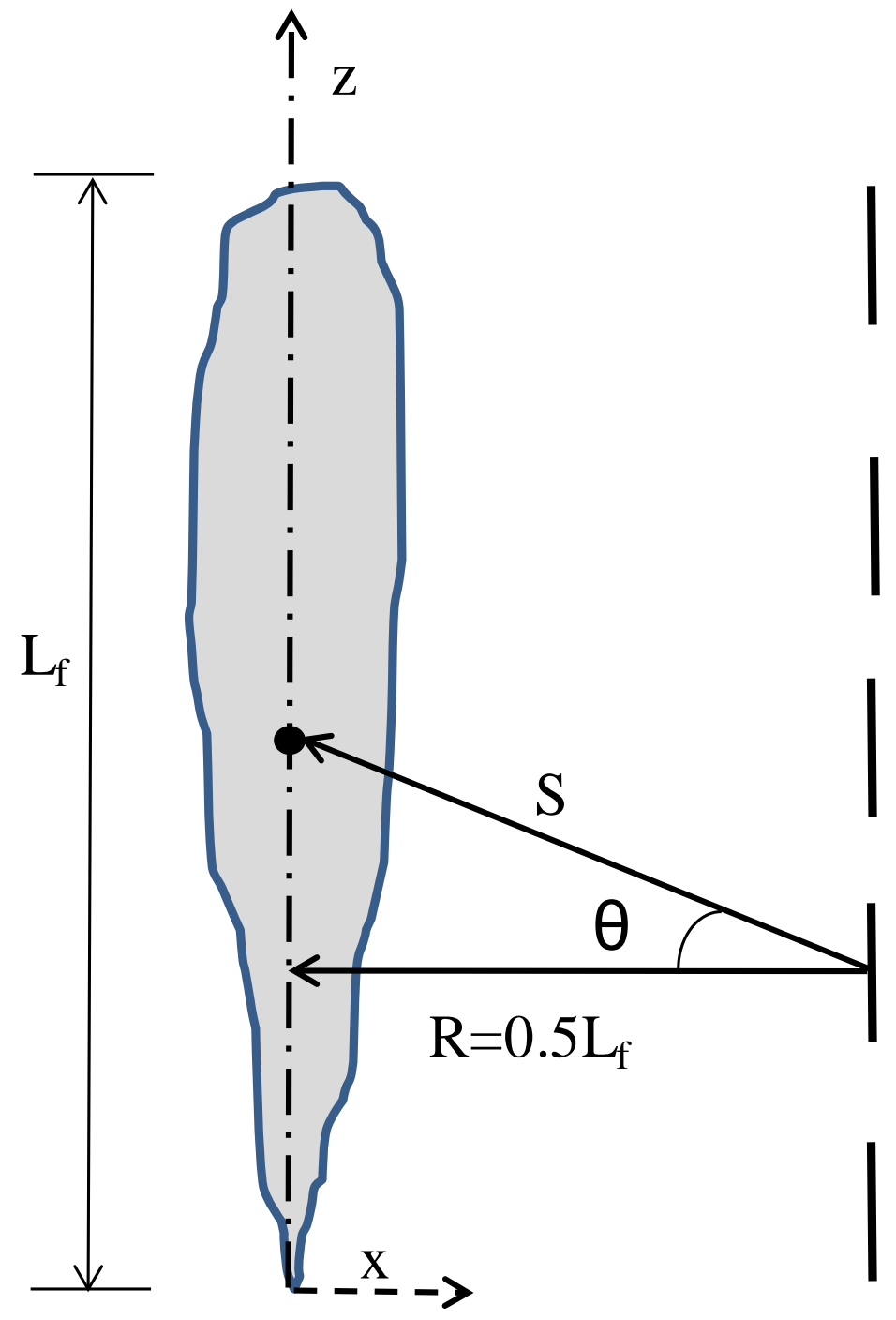

Fig.4: Experimental arrangement used by Sivathanu and Gore [16] 


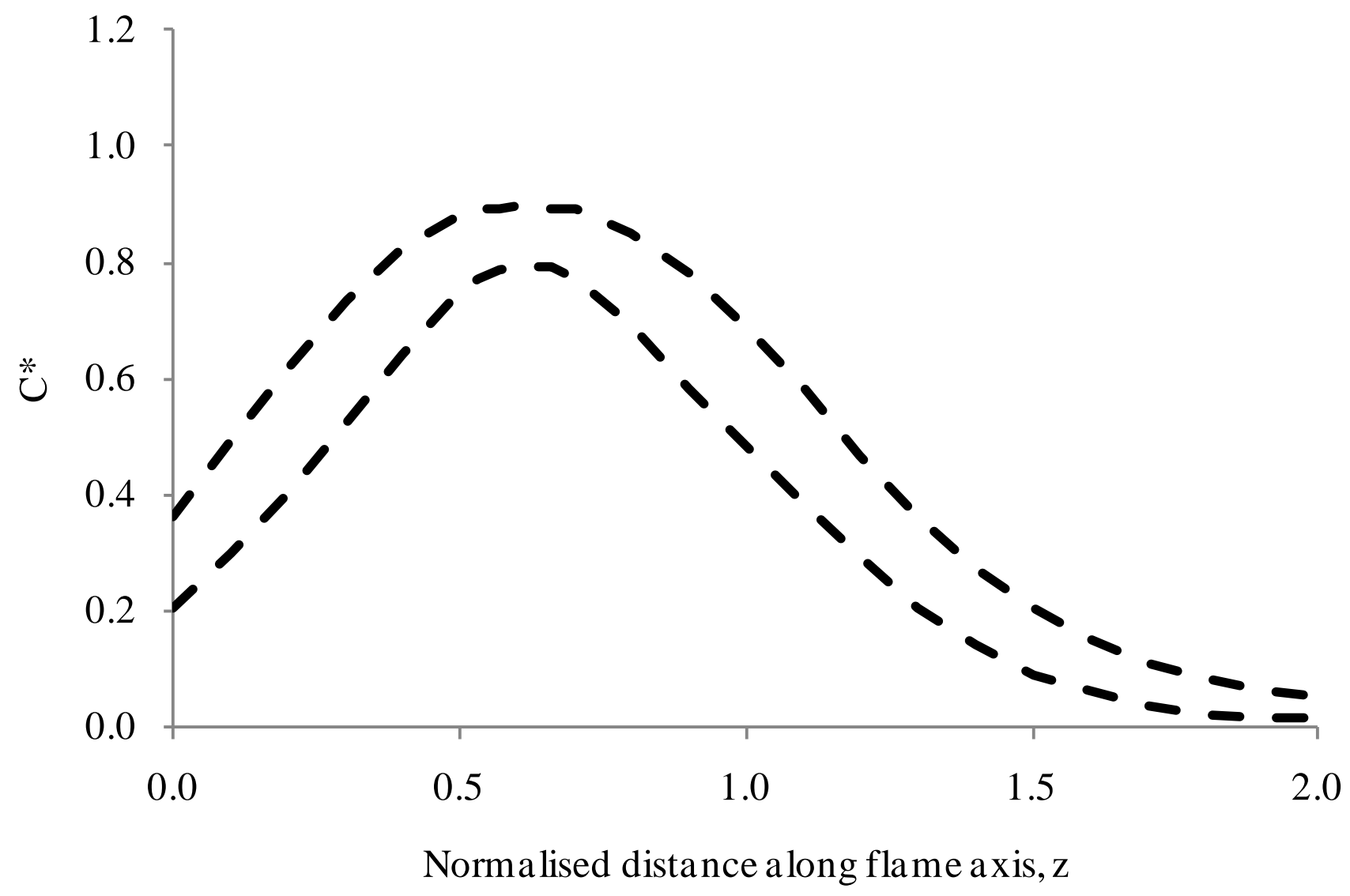

Fig.5: Bounds of data presented by Sivathanu and Gore [16] 


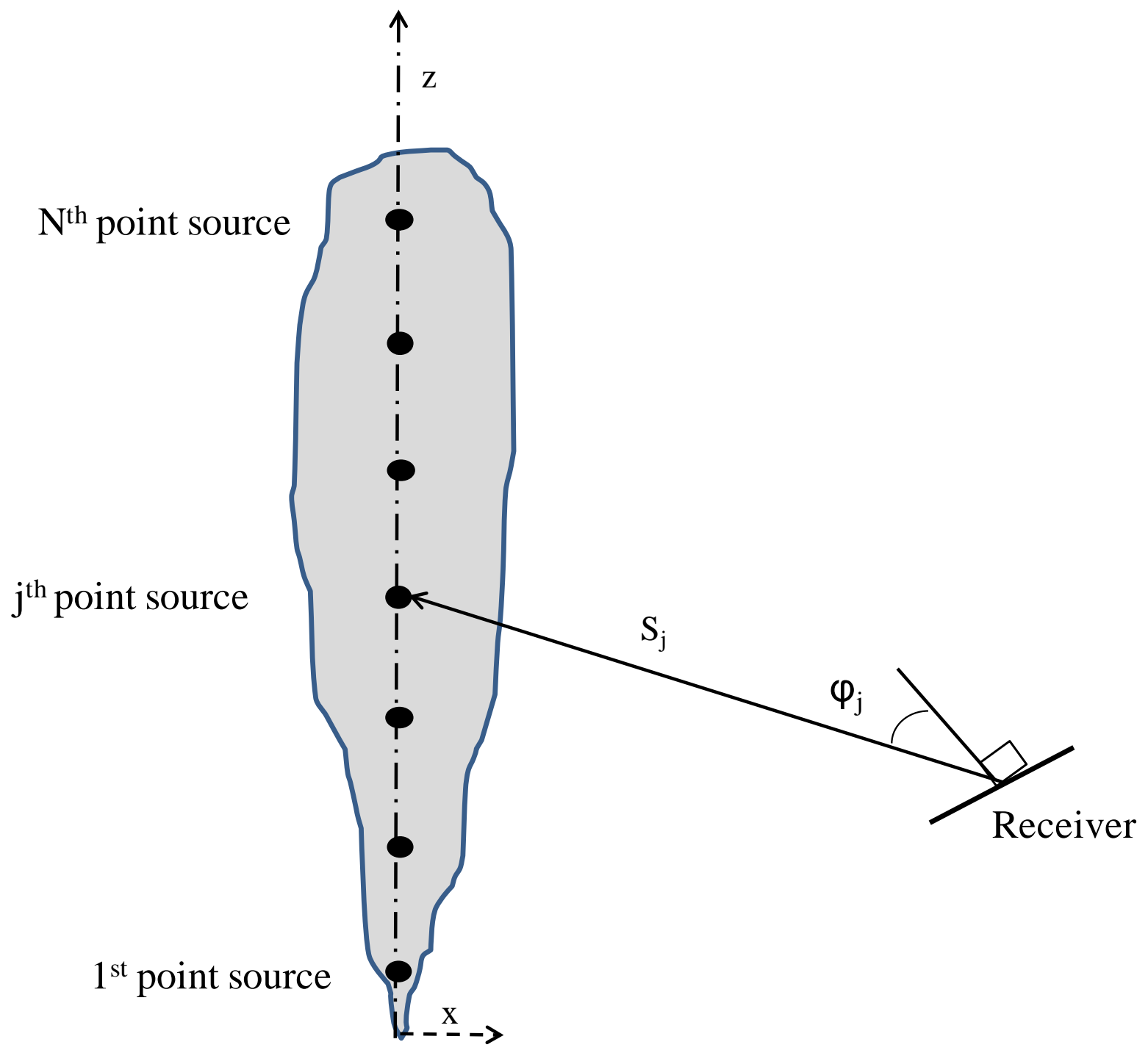

Fig.6: Multi-point Source model 


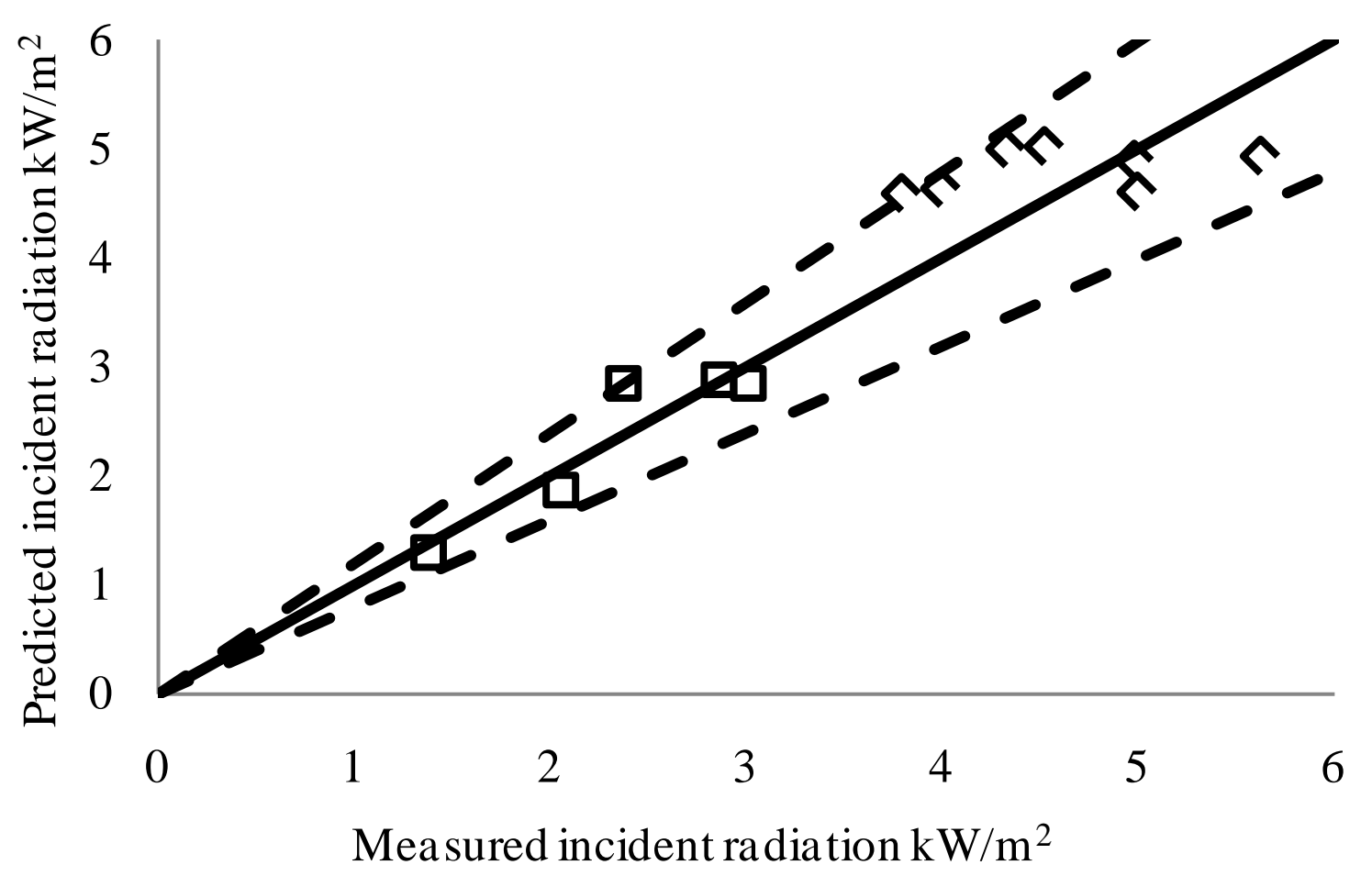

$$
\begin{array}{lll}
\curvearrowright & \text { Within } 1 \mathrm{Lf} & -\mathrm{Y}=\mathrm{X} \\
\boldsymbol{\square} & \text { More than 1 Lf } & -\mathrm{Y}=1.2 \mathrm{X} \text { and } 0.8 \mathrm{X}
\end{array}
$$

Fig. 7: Comparison of measured and predicted incident radiation using the weighted multi-point source model. Data from large scale natural gas jet fire (as yet unpublished) 


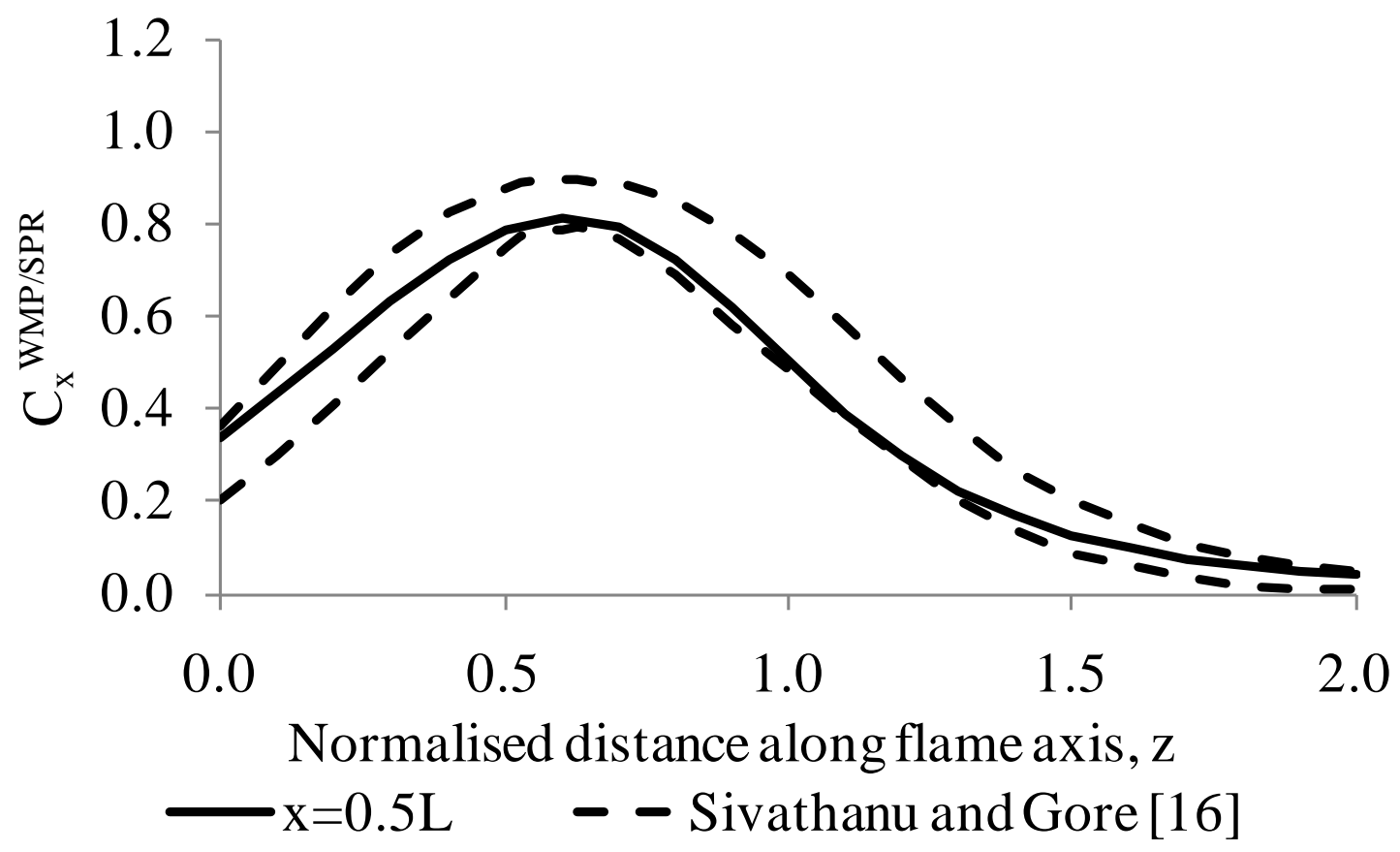

Fig.8: Predictions of $C_{x}^{W M P / S P R}$ compared with data of Sivathanu and Gore [16] 

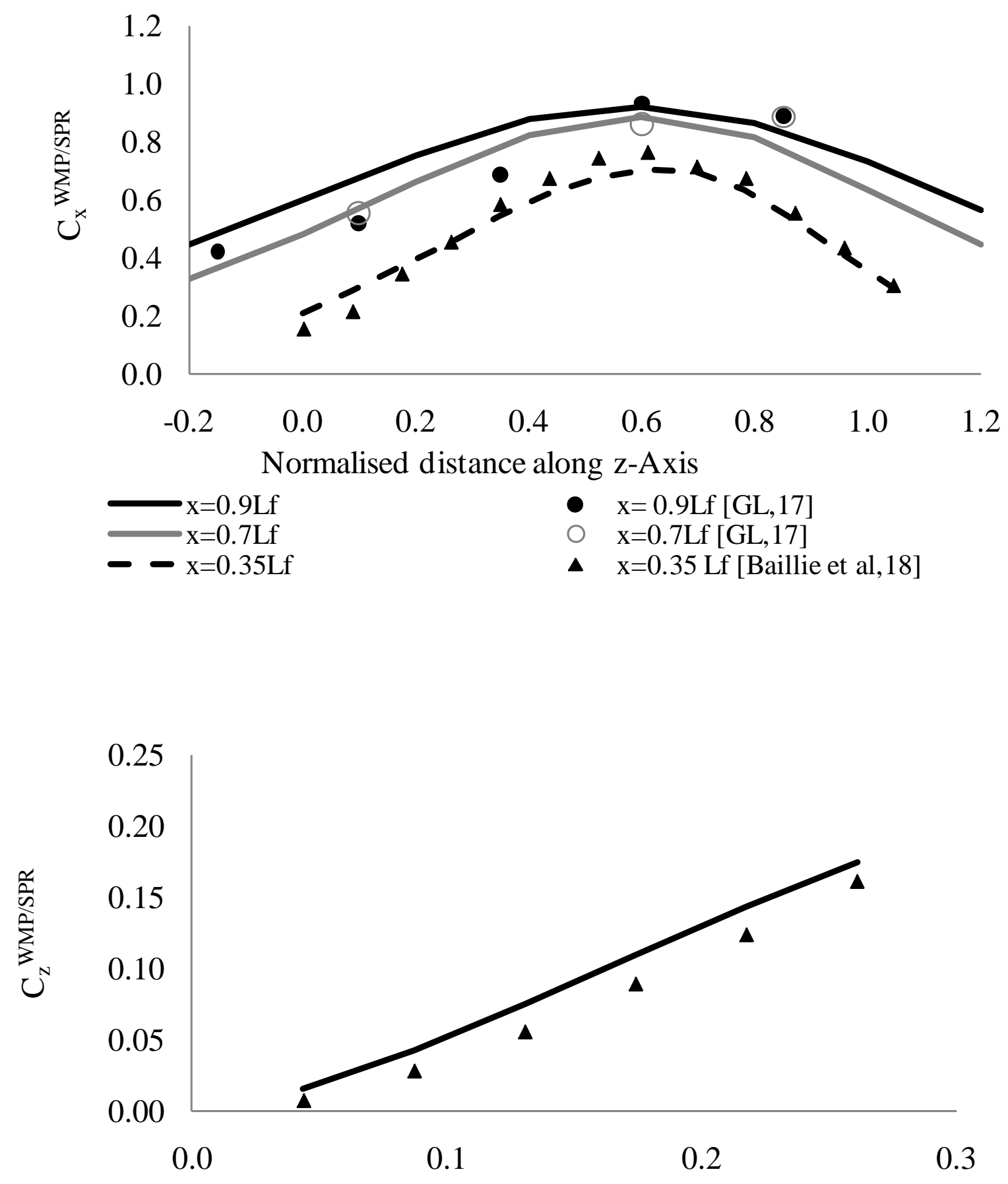

Normalised radial distance from flame axis, $\mathrm{x}$

Fig.9: Validation of WMP model in the near field by comparison with data: (a) $C_{x}^{W M P / S P R}$ for different radial distances; (b) $C_{z}^{W M P / S P R}$ for $\mathrm{z}=0$ 


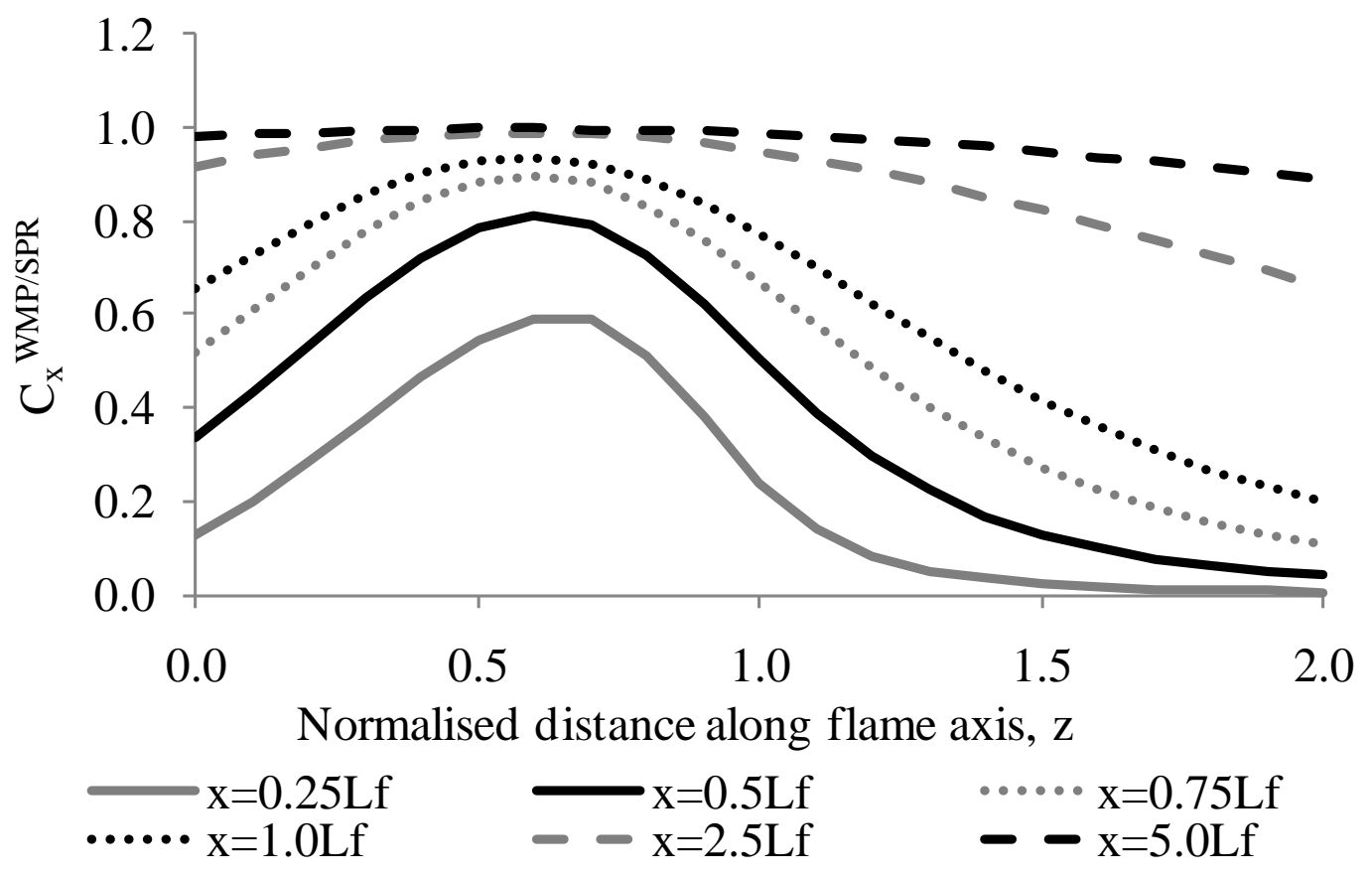

Fig.10: Predictions of $C_{x}^{W M P / S P R}$ for a range of radial distances 


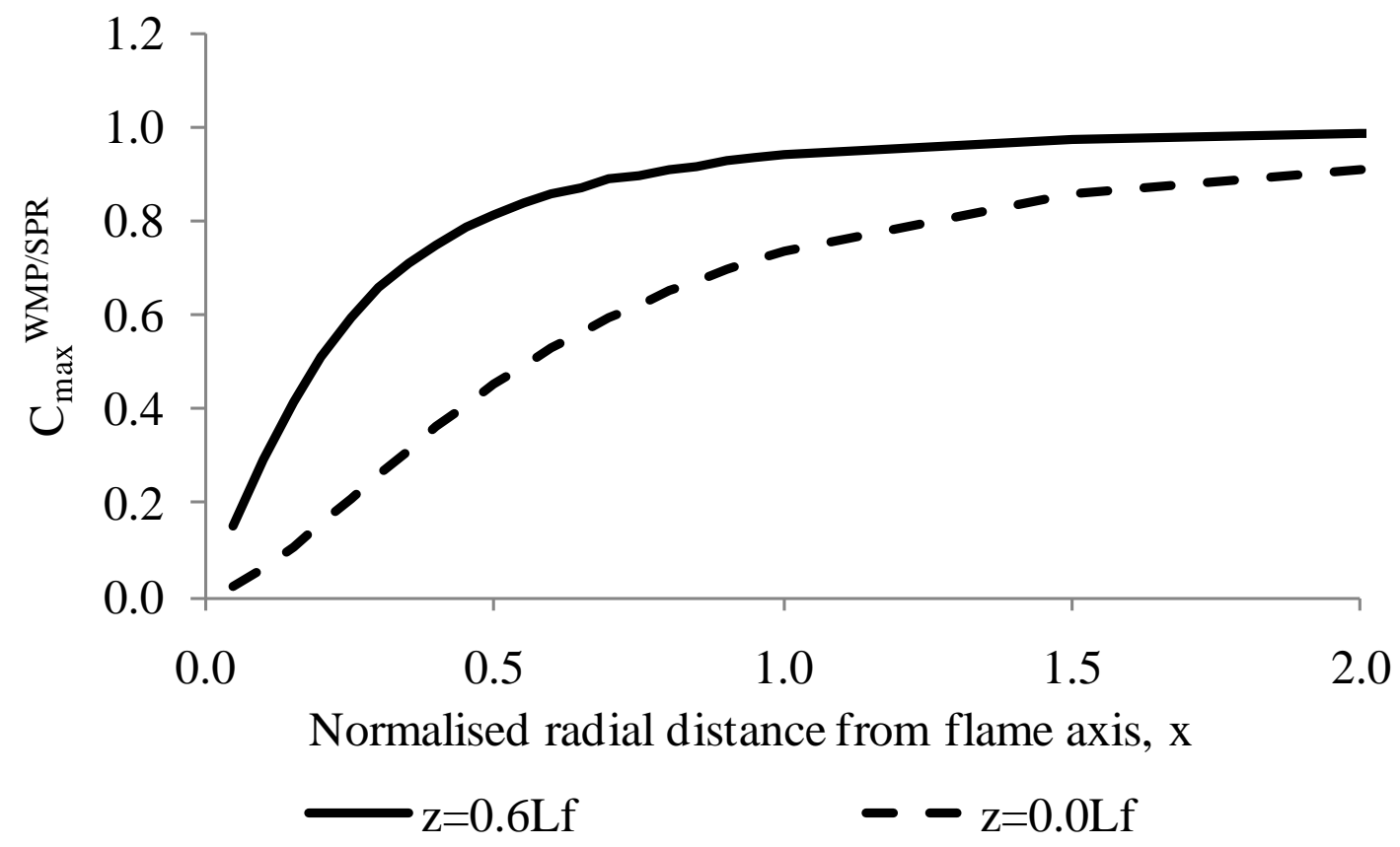

Fig.11: Predictions of $C_{\max }^{W M P / S P R}$ for two different axial positions 


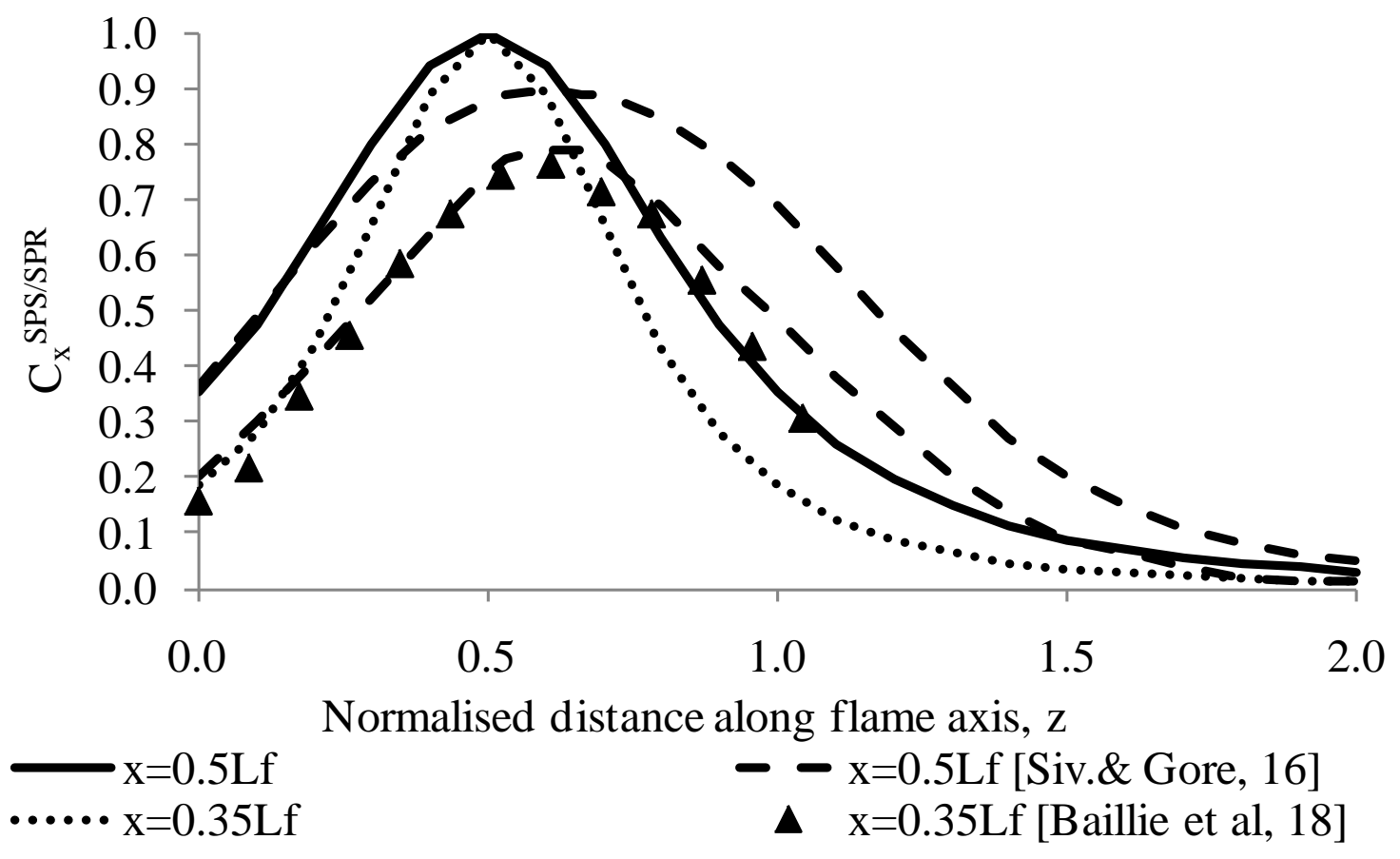

Fig.12: Predictions of $C_{x}^{S P S / S P R}$ compared with data for two different radial positions 


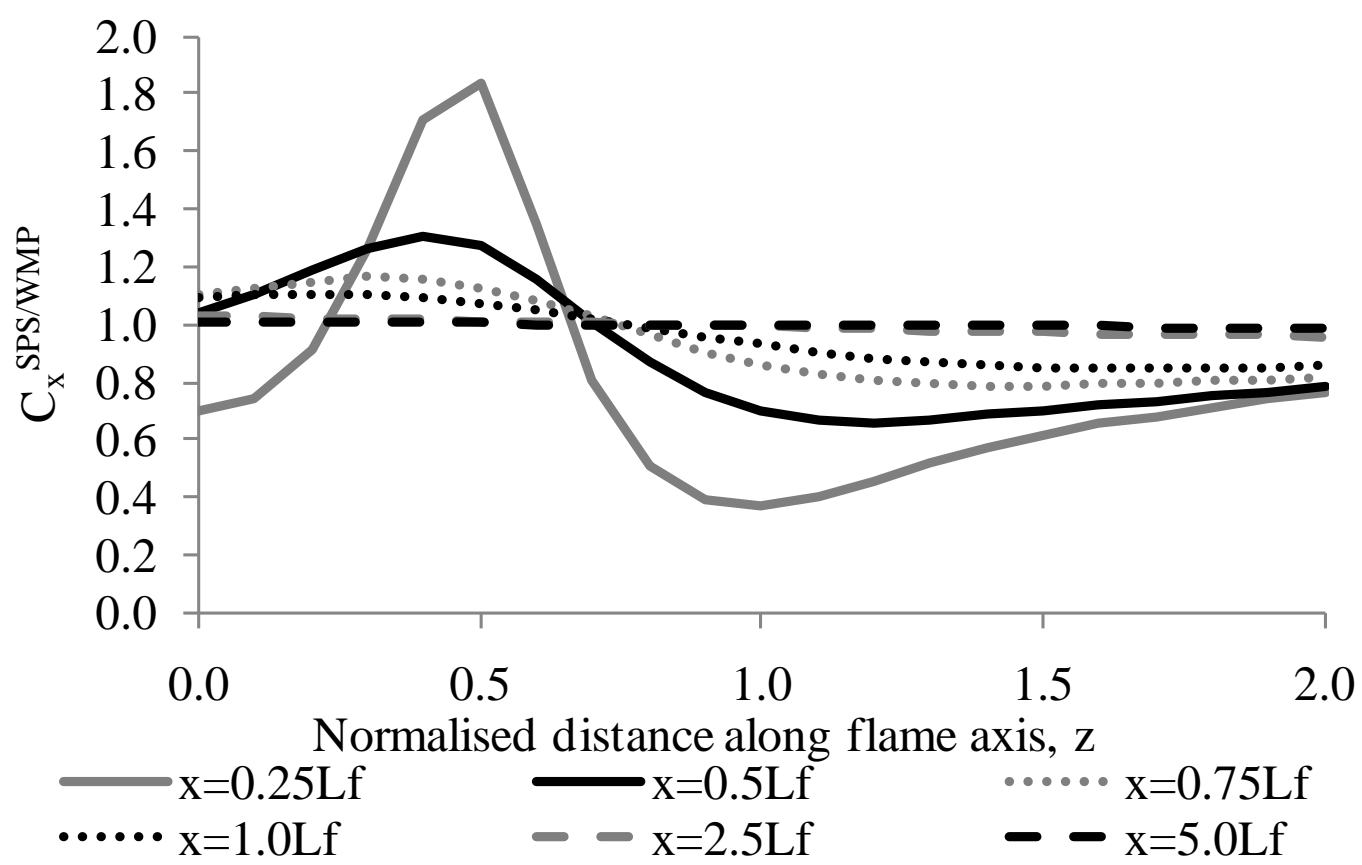

Fig.13: Predictions of $C_{x}^{S P S / W M P}$ for a range of radial distances 
(a)

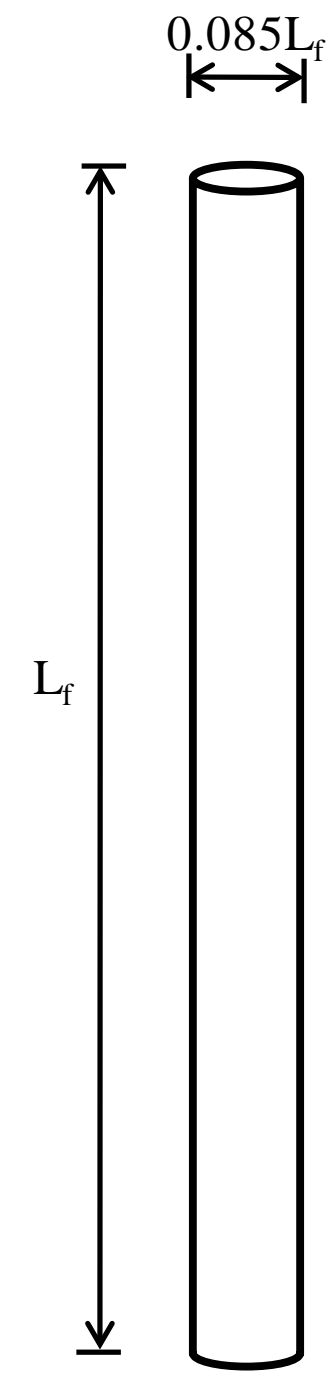

(b)
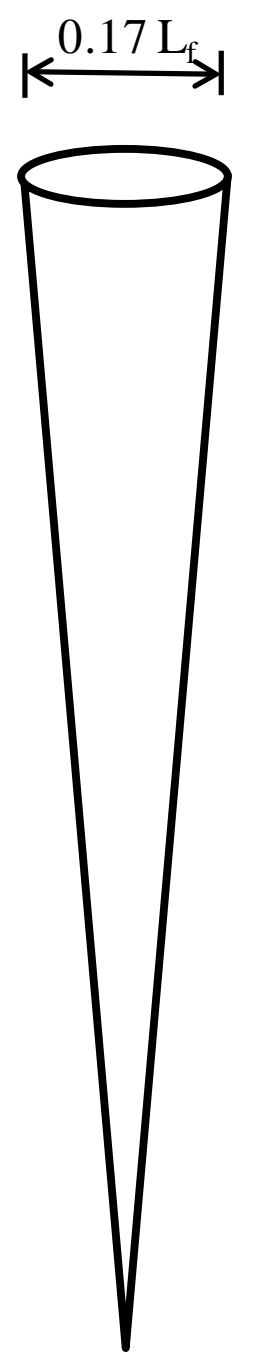

(c)
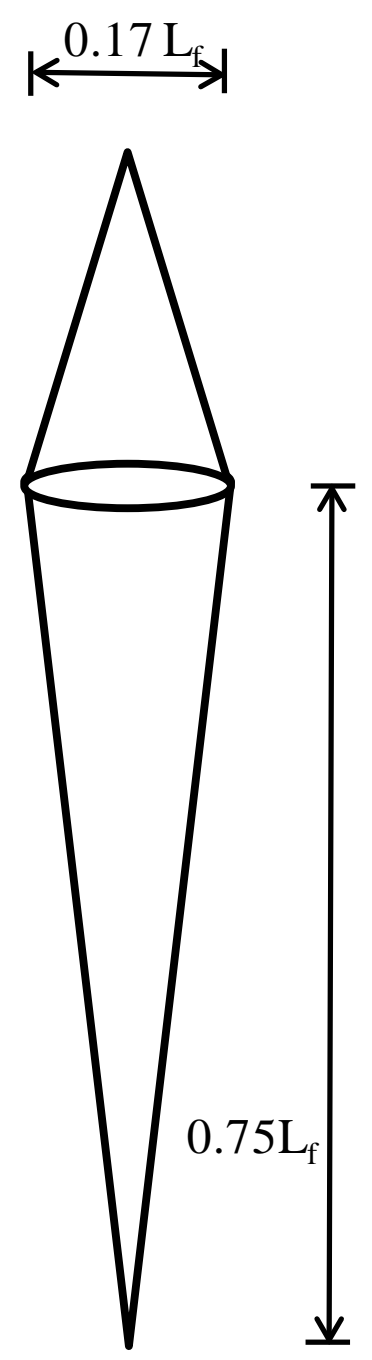

(d)
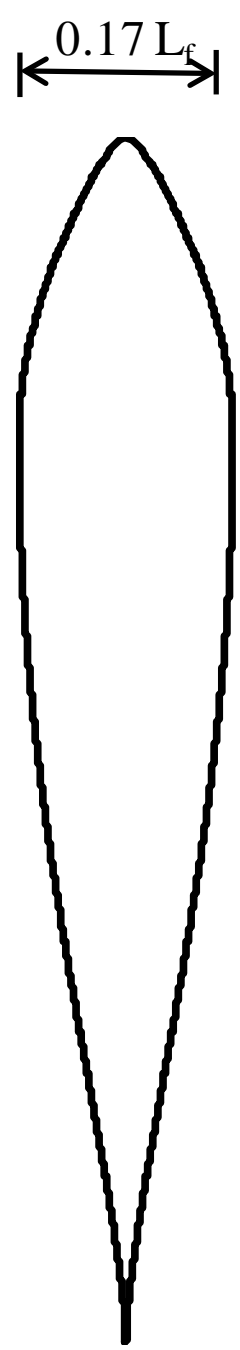

Fig.14: Solid flame shapes: (a) cylinder; (b) cone; (c) back-to-back cone; (d) back-to-back weighted ellipsoid. 


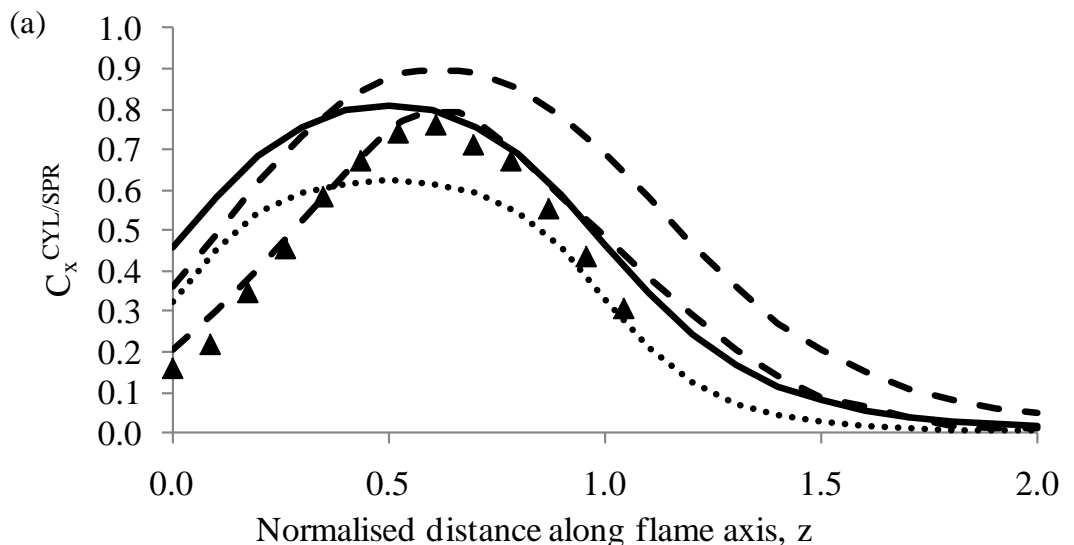

$\mathrm{x}=0.5 \mathrm{Lf}$

- $\mathrm{x}=0.5 \mathrm{Lf}[$ Siv. \& Gore, 16]

$\Delta \quad \mathrm{x}=0.35 \mathrm{Lf}$ [Baillie et al, 18]

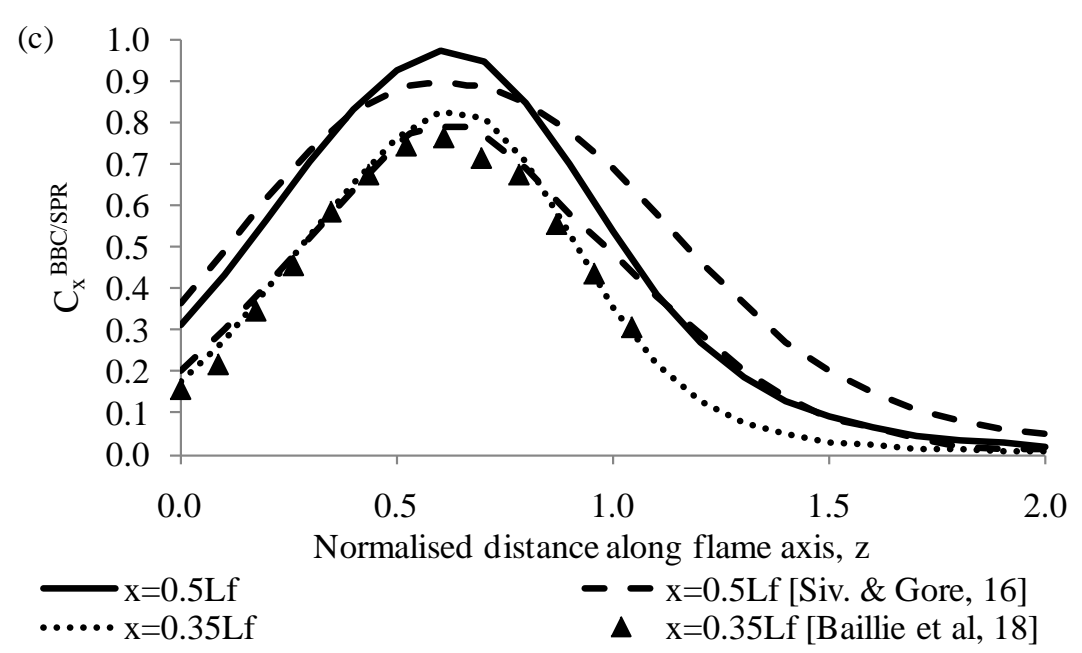

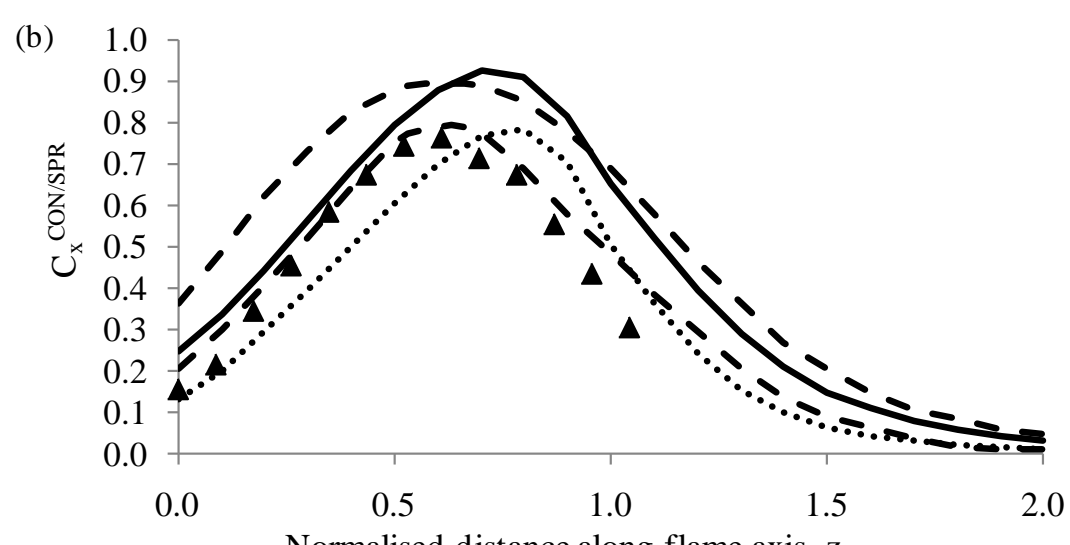

Normalised distance along flame axis, $\mathrm{z}$

- $\mathrm{x}=0.5 \mathrm{Lf}$ [Siv. \& Gore, 16]

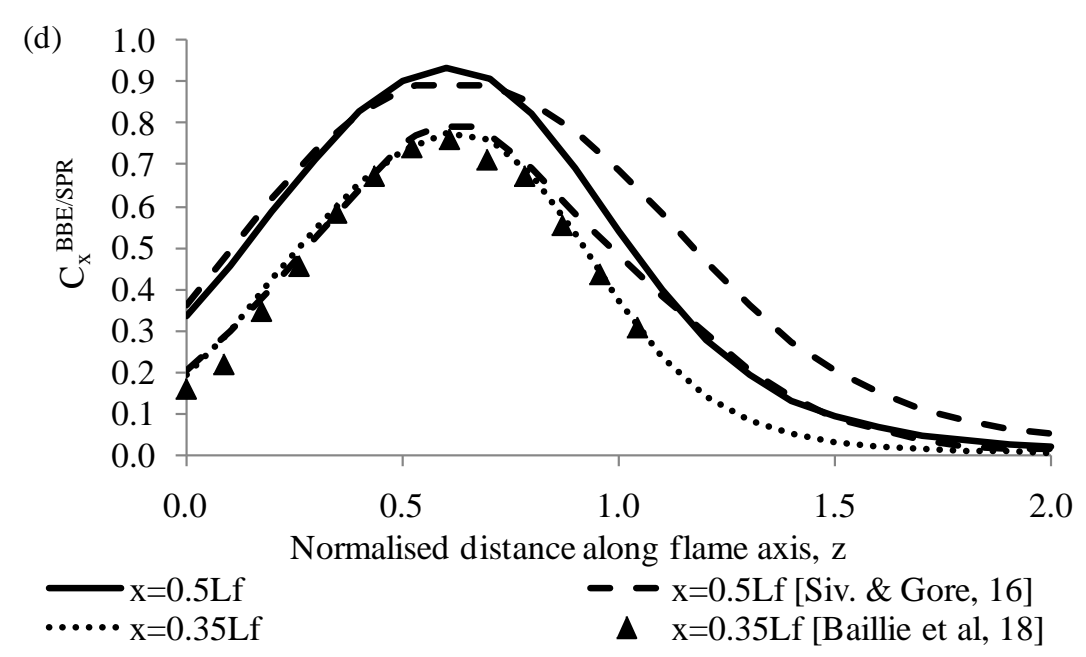

Fig.15: Predictions of solid flame models compared with data: (a) $C_{x}^{C Y L / S P R}$ from cylindrical flame model; (b) $C_{x}^{C O N / S P R}$ from conical flame model; (c) $C_{x}^{B B C / S P R}$ from back-to-back cone flame model; (d) $C_{x}^{B B E / S P R}$ from back-to-back ellipsoid flame model. 

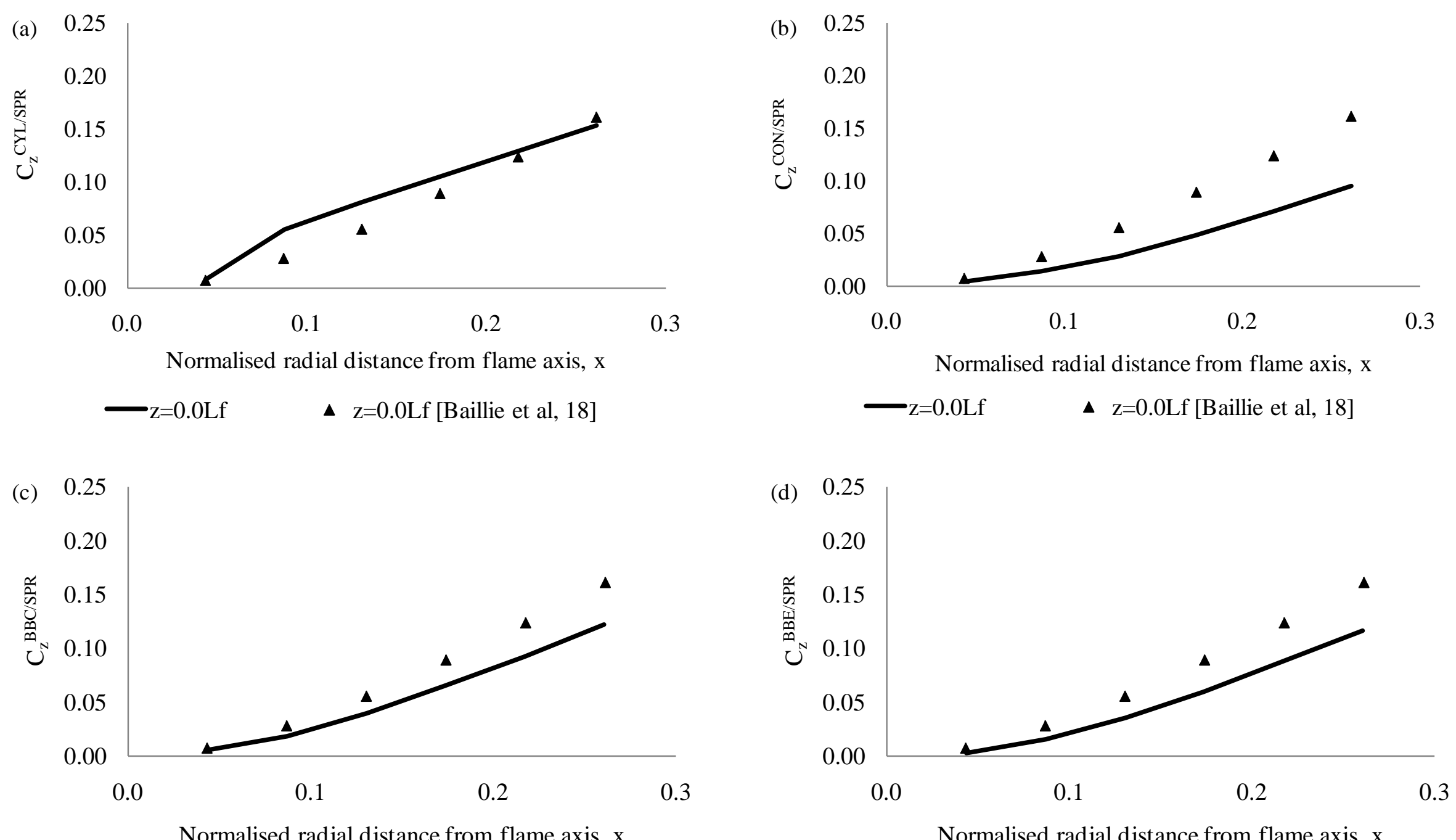

Normalised radial distance from flame axis, $\mathrm{x}$

- $\mathrm{z}=0.0 \mathrm{Lf} \quad \Delta \mathrm{z}=0.0 \mathrm{Lf}$ [Baillie et al, 18]

- $\mathrm{z}=0.0 \mathrm{Lf} \quad \boldsymbol{\mathrm { A }} \mathrm{z}=0.0 \mathrm{Lf}$ [Baillie et al, 18]

Fig.16: Predictions of solid flame models compared with data: (a) $C_{z}^{C Y L / S P R}$ from cylindrical flame model; (b) $C_{z}^{C O N / S P R}$ from conical flame model; (c) $C_{z}^{B B C / S P R}$ from back-to-back cone flame model; (d) $C_{z}^{B B E / S P R}$ from back-to-back ellipsoid flame model. 

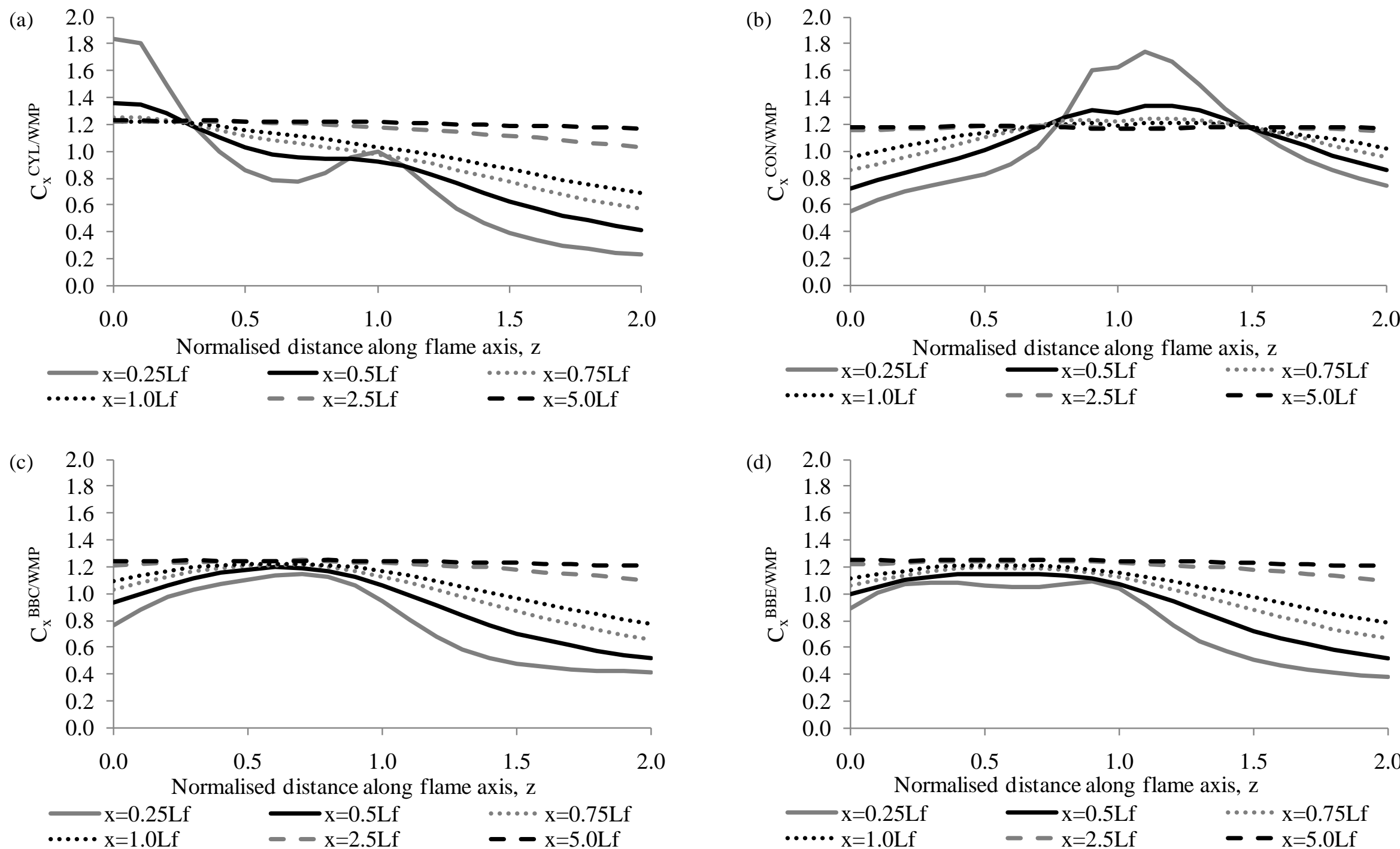

$$
\begin{array}{ccc}
x=0.25 \mathrm{Lf} & -\mathrm{x}=0.5 \mathrm{Lf} & \cdots \mathrm{x}=0.75 \mathrm{Lf}
\end{array}
$$$$
\text { …. } \mathrm{x}=1.0 \mathrm{~L}
$$$$
-\mathrm{x}=0.5 \mathrm{Lf}
$$$$
\text { - }-\mathrm{x}=5.0 \mathrm{Lf}
$$

Fig.17: Predictions of solid flame models for a range of radial distances of: (a) $C_{x}^{C Y L / W M P}$ from cylindrical flame model; (b) $C_{x}^{C O N / W P R}$ from conical flame model; (c) $C_{x}^{B B C / W M P}$ from back-to-back cone flame model; (d) $C_{x}^{B B E / W M P}$ from back-to-back ellipsoid flame model. 


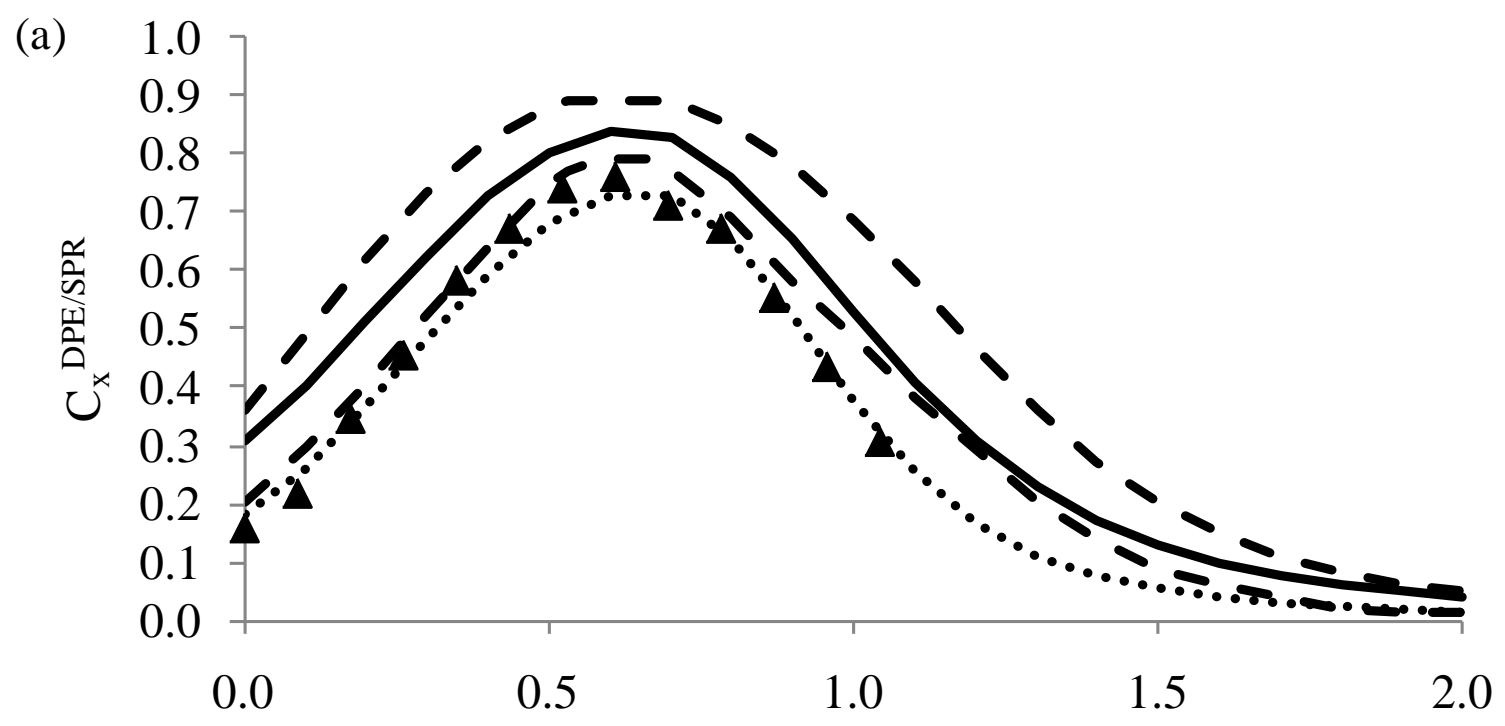

Normalised distance along flame axis, $\mathrm{z}$
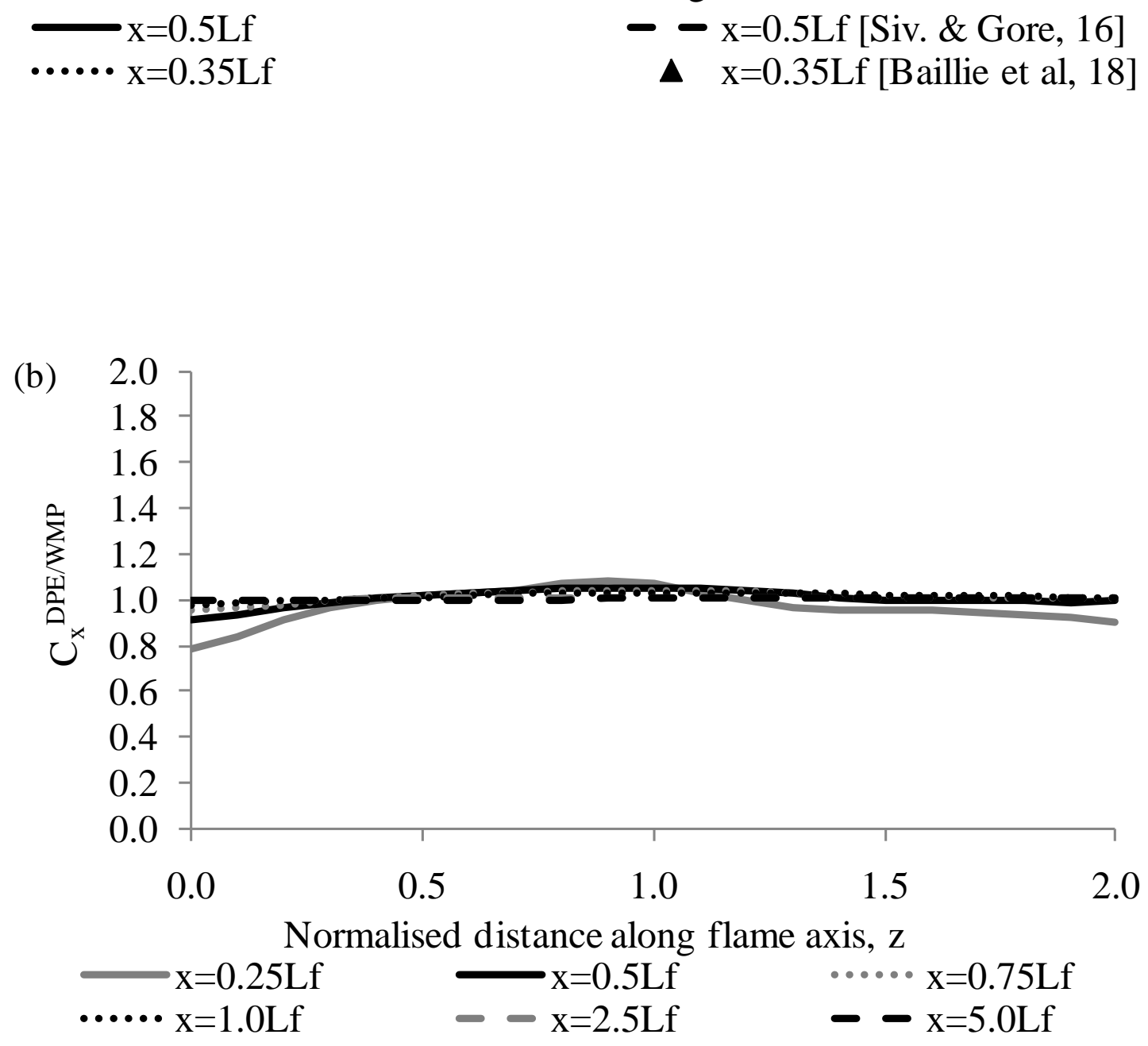


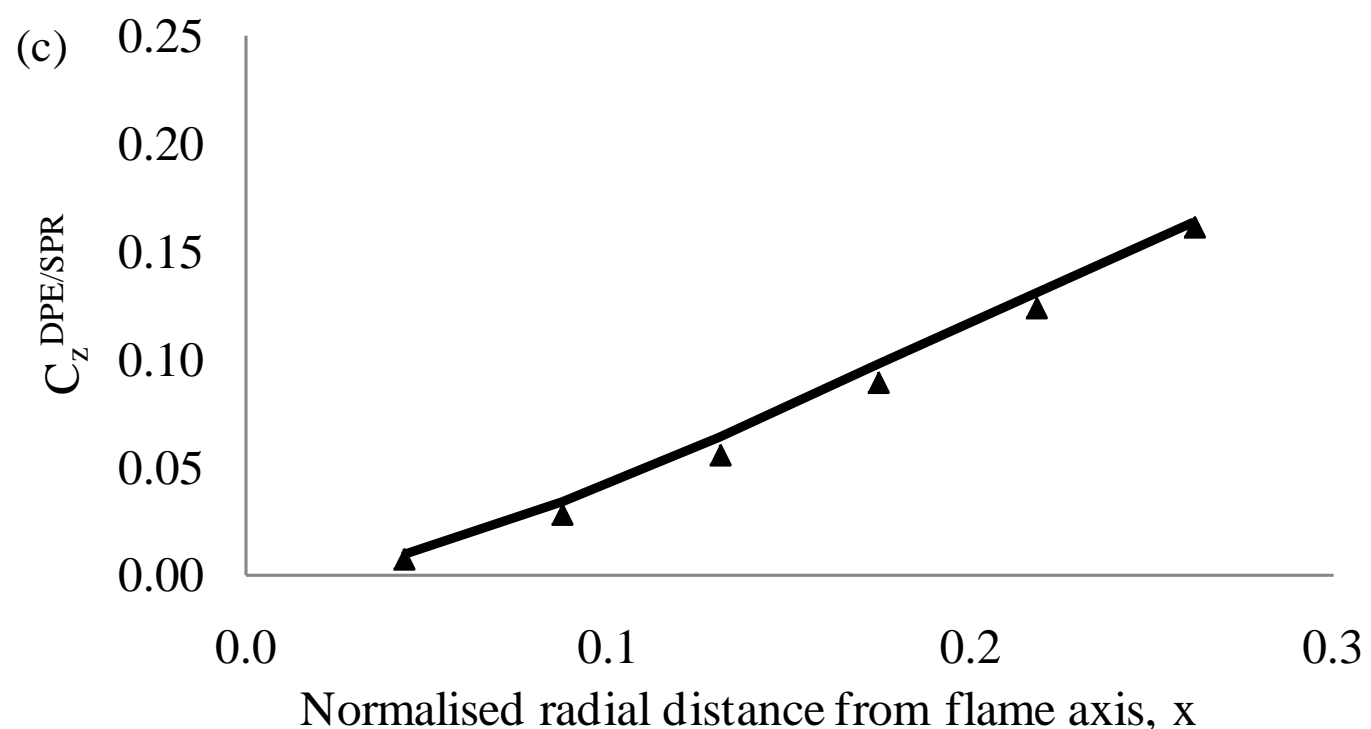

$-\mathrm{z}=0.0 \mathrm{Lf} \quad \boldsymbol{\mathrm { \Delta }} \mathrm{z}=0.0 \mathrm{Lf}$ [Baillie et al, 18]

Fig.18: Predictions of distributed point source model applied to the back-to-back weighted ellipsoid flame shape for: (a) two radial distances of $C_{x}^{D P E / S P R}$, (b) $C_{x}^{D P E / W P E}$. And (c) $C_{z}^{D P E / S P R}$ at $\mathrm{z}=0.0$ 